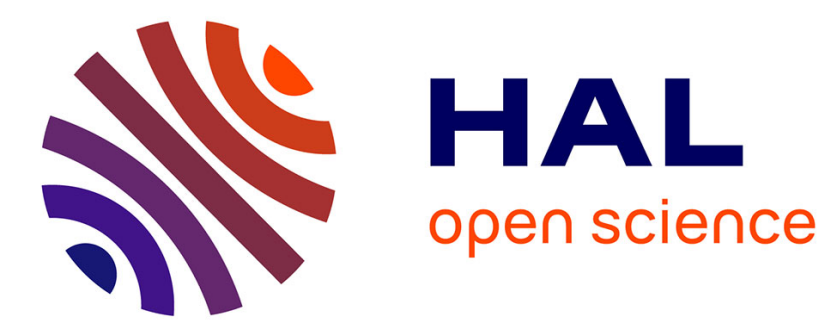

\title{
Geometrical properties of turbulent premixed flames and other corrugated interfaces
}

F. Thiesset, G. Maurice, F. Halter, Nicolas Mazellier, C. Chauveau, I. Gökalp

\section{To cite this version:}

F. Thiesset, G. Maurice, F. Halter, Nicolas Mazellier, C. Chauveau, et al.. Geometrical properties of turbulent premixed flames and other corrugated interfaces. Physical Review E , 2016, 93 (1), pp.013116. 10.1103/PhysRevE.93.013116 . hal-01660272

\section{HAL Id: hal-01660272 \\ https://hal.science/hal-01660272}

Submitted on 28 Mar 2019

HAL is a multi-disciplinary open access archive for the deposit and dissemination of scientific research documents, whether they are published or not. The documents may come from teaching and research institutions in France or abroad, or from public or private research centers.
L'archive ouverte pluridisciplinaire $\mathbf{H A L}$, est destinée au dépôt et à la diffusion de documents scientifiques de niveau recherche, publiés ou non, émanant des établissements d'enseignement et de recherche français ou étrangers, des laboratoires publics ou privés. 


\title{
On the geometrical properties of turbulent premixed flames and other corrugated interfaces
}

\author{
F. Thiesset, ${ }^{1}$ G. Maurice ${ }^{1}$ F. Halter,${ }^{1}$ N. Mazellier ${ }^{2}$ C. Chauveau,${ }^{1}$ and I. Gökalp ${ }^{1}$ \\ ${ }^{1}$ CNRS ICARE, Avenue de la Recherche Scientifique, 45072 Orléans Cedex 2 France \\ ${ }^{2}$ University of Orléans, INSA de Bourges, PRISME, EA 4229, 45072 Orléans, France
}

\begin{abstract}
This study focuses on the geometrical properties of turbulent flame fronts and other interfaces. In this purpose, we use an original tool based on Proper Orthogonal Decomposition (POD) which is applied to the interface spatial coordinates. The focus is mainly on the degree of roughness of the flame front which is quantified through the scale dependence of its coverage arclength. POD is first validated by comparing with the caliper technique. Fractal characteristics are extracted in an unambiguous fashion using a parametric expression which appears to be impressively well suited for representing Richardson plots. Then, it is shown that, for the range of Reynolds numbers investigated here, the scale-by-scale contribution to the arclength do not comply with scale-similarity, irrespectively of the type of similarity which is invoked. The finite ratii between large and small scales, referred to as finite Reynolds number effects, are likely to explain this observation. In this context, the Reynolds number that ought to be achieved for a proper inertial range to be discernible and for scale-similarity to be likely to apply, is calculated. Fractal characteristics of flame folding are compared to available predictions. It is confirmed that the inner cutoff satisfactorily correlates with the Kolmogorov scale whilst the outer cutoff appears to be proportional to the integral length-scale. However, the scaling for the fractal dimension is much less obvious. It is argued that much higher Reynolds numbers have to be reached for drawing firm statements about the evolution (or constancy) of the fractal dimension with respect to flame/flow parameters. Finally, a heuristic phenomenology of corrugated interfaces is highlighted. The degree of generality of the latter phenomenology is confirmed by comparing the folding of different interfaces including a turbulent/non turbulent interface, a liquid jet destabilized by a surrounding air jet, a cavitating flow, and an isoscalar evolving in a turbulent medium. The latter outcome is likely to have strong implications for modelling the corrugation of turbulent interfaces occuring in many physical situations.
\end{abstract}

PACS numbers: 47.70.Pq, 47.27.-i, 47.53.+n, 47.55.Ca

\section{INTRODUCTION}

The laminar flamelet concept [1] is without doubt the most utilized hypothesis for describring turbulent premixed flames, primarily because of the resulting analytical and modelling simplifications. Under this assumption, turbulent wrinkled flames are treated as a collection of thin layers, whose inner structure is identical to a (possibly stretched) one-dimensional laminar flame, propagating normal to themselves in the direction of the unburned turbulent mixture. As a consequence, the notion of laminar flamelet implies that only the geometrical properties of the flame (its surface) are needed for accurately predicting macroscopic features such as the global consumption speed or heat release. This explains the incontestable success of the so-called geometrical approaches for closing the transport equations of e.g. temperature or progress variable in either RANS (Reynolds Averaged Navier-Stokes) or LES (Large-Eddy Simulation) context. According to Veynante and Vervisch [2], these geometrical methods include the G-field equation [3$5]$, the flame surface density approach [6-8], and the flame wrinkling description (see [9-11] among others). It is also worth stressing that because of the analytical bridges that were highlighted in [2] between the latter approaches and other methods such as the PDF or the scalar dissipation rate closures, the reliability of all models finally depend more or less directly on our ability to describe the geometrical properties of the turbulent flame front.

However, predicting the evolution of the flame surface in a turbulent flow is far from being an easy task. To a great extent, the progress in our understanding of the geometrical aspects of turbulent flames has been hindered by our inability to finely describe and predict their multi-scale nature. In effect, flame wrinkling exhibits a wide and continuous range of scales, and suggests it is rather difficult to characterize and quantify the surface without any appropriate analytical means. The multi-scale facet of corrugated flamelets arises from the interaction that the flame experiences with turbulence, which itself features a wide range of turbulent eddies. These extend from the integral length-scale, which retains through its dynamic and topology significant informations about initial/boundary conditions [12], to the inertial and dissipative range down until the Kolmogorov lengthscale for which a universal statistical description is assumed [13].

Whilst in the context of RANS the total flame surface is needed for closing the transport equations, in LES, only the unresolved flame surface, i.e. the flame surface embedded in a volume of size smaller than the mesh grid, is required. As a consequence, one has to tackle a local (sometimes referred to as a scale-byscale) description of flame folding by focusing on the contribution of each scale of size $r$ to the interface 
folding. This appears quite natural notwithstanding the aforementioned multi-scale facet of turbulent flames. In addition, by analogy with the turbulent velocity field for which the smallest scales have the best prospect of being universal [13, 14], the smallest scales of flame wrinkling might also exhibit similar attributes. This notion of scale similarity or scale universality states that the scale-by-scale distribution of a fluctuating quantity (i.e. as a function of the scale $r$ ) can be universal when scaled by an appropriate set of similarity variables. This feature is worth being validated for turbulent flames since it suggests that a universal subgrid-scale model for the flame wrinkling might be derived. This encompasses investigating in detail the degree with which the flame folding at a given scale complies with scale-similarity and emphasizing the associated relevant normalizing scales. To our knowledge, this has never been done.

Among the methods that allows to quantify the scale-by-scale contribution to the flame wrinkling or more generally rough interfaces, fractal analysis is one of the most common. Fractals first originate from Richardson and later from Mandelbrot who built up the mathematical framework for describing the scaledependence of the surface roughness. Since then, it has been applied to an extremely wide variety of physical situations. Pragmatically speaking, a fractal behaviour is identified when coarse graining the rough interface at a scale $r$ results in a power-law dependence of its surface of the form $r^{-\beta}$. The power-law exponent, $\beta$ is referred to as the fractal dimension, which provides a measure of the degree of roughness of such an interface. For instance, the fractal dimension of a smooth surface evolving in a volume tends to 2 whereas it approaches 3 when the surface fills the entire domain.

Gouldin $[15,16]$ was first to apply fractal concepts to turbulent flames, and since yet, there has been wealth of experimental [17-20], numerical [9, 11, 21] and analytical $[22,23]$ support in favour of a fractal power-law variation for the distribution of flame folding as a function of the scale $r$. Whilst fractal models appear to be well suited for modelling the flame surface density, it still requires some efficient sub-models for predicting the fractal characteristics as a function of the local flame/flow parameters. For instance, in LES, the fractal dimension is often estimated using a dynamic approach $[10,11,24]$ following the lines of Germano et al. [25]. The systematic improvement of the simulation results when such a procedure is invoked suggests that the current predictions for the fractal characteristics fail. In other words, it indicates that we still misunderstand the physical processes at play and that more work is needed in order to give further insight into the phenomenology of flame wrinkling with the aim of providing reliable predictions for the fractal characteristics.

Beyond turbulent combustion, there are several phenomena than can be described in terms of wrin- kled surfaces evolving in a turbulent flow [6]. These phenomena include for instance turbulent mixing and two-phase flows, which are widely encountered in a very large variety of combustion applications. Their understanding is thus of primordial importance. Following Pope [6], all these types of interface can be classified into (i) material surfaces, which pertain to e.g. two phase-flows where the interface is often tracked using a level-set equation [26], (ii) constantproperty surfaces for tackling e.g. passive scalar mixing, diffusion flames [6], and (iii) propagating surfaces which have been widely applied to e.g. premixed flames $[3,4,27]$. Also mentioned in [6], these three types of surfaces can be regarded as constantproperty surfaces or as propagating surfaces. This has led Peters [4] to unify the analytical description of premixed flames for both the corrugated flamelet regime and the thin reaction zone regime. The aforementioned analogy between different types of interfaces probably explains why fractal concepts successfully apply to all of these. It is in effect well known that turbulence and turbulent mixing (see e.g. [2832] among others), two-phase flows [33-35], material lines evolving in turbulent flows [36] reveal some degree of fractality. In addition, the surface density concept which, as mentioned before, is widely used in the field of turbulent combustion, applies also in the context of turbulent mixing [37] and two-phase flows [38]. In other words, all these different phenomena can be described via their geometrical properties. It is also worth stressing that, speculatively, all these interfaces might further reveal some degree of similarity or universality notwithstanding their common transport equation [6]. One particular aspect of the present study is to give further insight into the analogy of the geometrical aspects of different turbulent interfaces.

In previous studies, the fractal characteristics of turbulent interfaces were generally obtained by use of the box-counting method or the caliper technique. In the present study, Proper Orthogonal Decomposition (POD) is used as an alternative method. The POD, also known as a Karhunen-Loève decomposition or principal component analysis, is a data-driven modal decomposition that has become very popular for investigating the non-linear processes in physics, in particular [39-41]. It has been generally applied to the velocity field, with the aim of extracting typical spatial and/or temporal patterns of the coherent large scales in turbulent flows [41, 42]. POD is usually based on the two-points correlation which is computed from either long-time series or large realizations ensemble. Statistical stationnarity ensures the two-points correlation and accordingly the empirical function basis to be weakly sensitive to the database.. In the field of combustion, very few studies have focused on modal decompositions. POD has been used by Danby et al. [43] to investigate the auto-ignition process of inhomogeneous hydrogen air 
mixture. These authors carried several preprocessing techniques on the data-set in order to optimize the number of modes needed to minimize the reconstruction error. Later, using Particle Image Velocimetry data sets and $\mathrm{OH}-\mathrm{Planar}$ Laser Induced Fluorescence together with the extended POD method introduced in [42], Duwig \& Fureby [44] and Duwig \& Fuchs [45] used POD to study (i) thermo acoustics instabilities, (ii) the interaction of precessing vortex core with a swirling flame and (iii) unsteady flames driven by acoustic perturbation. Finally, to characterize the flame shedding process behind a bluff-body, Kostka et al. [46] used POD and flame chemiluminescence images to educe coherent behaviours and separate the energy at given mode into symmetric, asymmetric, and uncorrelated components. Here, we propose to apply the POD algorithm to the spatial coordinates of the flame contours with the aim of extracting quantitative informations on the fractal characteristics of flame folding [47]. To the best of our knowledge, this has never been undertaken before. Note also that other data-decomposition than POD exists. One of these is the Dynamic Mode Decomposition (DMD) which was derived independently by Rowley et al. [48] and Schmid [49]. However, the latter method invokes a linear mapping between two time consecutive fluctuating events. Consequently, this type of decomposition which is limited only to time-resolved numerical or experimental data is not tractable in our case, where part of the measurements were made using low frame rate Mie scattering tomography. Bidimensional Empirical mode decomposition (EMD) $[50,51]$ could also have been used. Nevertheless, the number of modes that arises from this decomposition is generally rather limited. This means that the spectral precision of the latter decomposition is not reliable enough for accurately quantifying the scale dependence of the flame wrinkles.

On the basis of an extensive experimental study using Mie scattering tomography measurements in a turbulent Bunsen Burner, the present paper addresses to the following issues. (i) Is the POD reliable for extracting quantitative informations on the geometrical properties of turbulent interfaces? How does it compare to other methods such as the caliper technique? (ii) Does the distribution of wrinkling as a function of $r$ comply with scale-similarity? If yes, what are the relevant normalizing scales? (iii) What is the ability of some existing empirical or phenomenological relations for predicting the fractal characteristics of corrugated flames? (iv) Are there similar patterns in the folding distribution of different interfaces? Could they be represented by a unique heuristic phenomenology?

The paper is organized as follows. In section $\S$ II A, the experimental apparatus and measurement technique are presented. A comprehensive list of parameters pertaining to the present database consisting of 24 operating conditions is also reported. Spe- cial emphasis is further given to the application of the POD algorithm to the flame front spatial coordinates $(\S I I \mathrm{~B})$. Then, the geometrical properties of the turbulent flames are investigated in detail in $\S$ III. Comparison with the caliper technique is presented in $\S$ III A together with the results pertaining to the scale-similarity analysis (§III B). The evolution of the fractal properties of the turbulent flames are discussed and compared to available theoretical or empirical predictions in $\S I V$. Finally, the scale-byscale distributions of wrinkling of different turbulent interfaces including turbulent flames, passive scalar iso-values, a turbulent/non turbulent interface and a two-phase and a cavitating flow are presented in $\S \mathrm{V}$. Conclusions are drawn in $\S \mathrm{VI}$

\section{EXPERIMENTAL SETUP AND DATA ANALYSIS}

Before focusing on the scale-by-scale properties of the turbulent flame fronts, we first turn our attention to the description of the experiments and data analysis that have been undertaken in the present study.

\section{A. Experimental setup and description of the database}

Experiments were carried out in a high pressure Bunsen burner (Fig. 1). An earlier version of this burner originated from Ref. [52] was recently upgraded by Refs. $[53,54]$ by replacing the mono-grid turbulence generator by a multi-scale grids generator [55] with the aim of achieving a higher turbulent intensity. The Bunsen burner (see Fig. 1) has a diameter of $25 \mathrm{~mm}$ surrounded by annular co-flow of inner and outer diameter of $30 \mathrm{~mm}$ and $50 \mathrm{~mm}$ respectively. Flames are stabilized with the help of a stoichiometric methane/air pilot annular flames located between the inner burner and the co-flow. The annulus is $2.5 \mathrm{~mm}$ wide. The burner is placed in a pressure chamber of inner diameter $300 \mathrm{~mm}$ and height $1350 \mathrm{~mm}$ that allows to reach pressure magnitudes up to $0.5 \mathrm{MPa}$. The multi-grid turbulence generator consists of 3 consecutive grids with different mesh size $(2,5,12.5 \mathrm{~mm}$ from up- to downstream) and different solidity (46, 57 and $67 \%$ ) placed in a way that maximizes the turbulence energy production [55]. The gap between the first (upstream) and second grid is $7 \mathrm{~mm}$ while the second and third grid are separated by a distance of $17 \mathrm{~mm}$. The last grid is located $60.5 \mathrm{~mm}$ upstream the burner exit to ensure the flow to be statistically homogeneous in the transverse direction. One- and two-points statistics of the velocity field have been inferred from extensive measurements using both hotwire and Laser Doppler Velocimetry [53]. These measurements indicate that although the decay of turbulent kinetic energy is faster for the multi-grid case, 


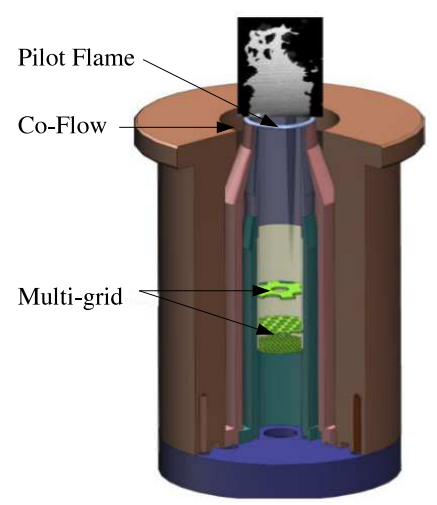

FIG. 1. (Color online). High-pressure Bunsen burner with the multi-grid injection system used in the present study

the turbulence intensity is roughly doubled by comparison with the mono-grid case. In addition, the turbulent small scales are smaller and contain more energy [53].

Measurements are carried out by means of both low $(10 \mathrm{~Hz})$ and high $(10 \mathrm{kHz})$ frame rate Mie-scattering tomography. (i) For the low frame rate tomography, a Nd-Yag laser 532nm and a CCD camera (PIV TSI 4M, $2048 \times 2048$ pixels $^{2}$ ) equipped with a $105 \mathrm{~mm}$ F2.8 lens were used. In this case, $N_{i m}=1000$ images were recorded. The resolution was in the range 0.035 $0.075 \mathrm{~mm} / \mathrm{px}$ depending on the case. (ii) For the high frame rate tomography, use was made of a continuous Coherent Verdi G20 Laser which delivers up to $20 \mathrm{~W}$ at $532 \mathrm{~nm}$. The light scattered by the particles is then captured by a Phantom V1210 camera, equipped with a $105 \mathrm{~mm} \mathrm{F2.8} \mathrm{lens,} \mathrm{working} \mathrm{at} \mathrm{an} \mathrm{ac-}$ quisition rate of $10 \mathrm{kHz}$ with a field of view of $800 \times 384$ pixels ${ }^{2}$. $N_{i m}=10^{4}$ images were recorded and the resolution was $0.108 \mathrm{~mm} / \mathrm{px}$. In both case, seeding of the flow is made with silicon oil droplets supplied by an atomizer. Typical size of droplets is about $1 \mu \mathrm{m}$. The effect on the chemical reactions of adding oil droplets is still an open question. Here, we consider that the amount of oil which is added is sufficiently small for not modifying the global flame properties such as the laminar flame speed and thickness.

The flame contour is then extracted as follows. Firstly, a contrast-limited adaptive histogram equalization (CLAHE) is applied to the original images in order to optimize the contrast in the images. Then, to limit the pixelixation associated with the CLAHE, images are filtered using a Gaussian filter of size equal to 4 times the spatial resolution. For the binarizing procedure, we use a standard threshold-based technique. More precisely, the histogram of the gray scale is calculated. The latter reveals two distinct peaks corresponding to the fresh and burned gas respectively. The threshold value for discriminating the flame contour is set as the average value between the gray scale of these two peaks. Yields estimations for the progress variable, noted $c$, which is by definition

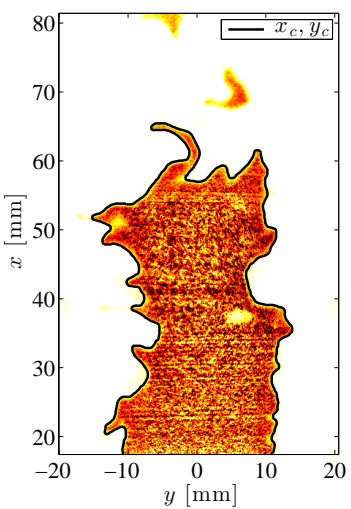

FIG. 2. (Color online). Typical image showing the reliability of the contour extraction and filtering for Flame 18 (Table I). The black line is the contour coordinates while the colormap shows the light intensity recorded by the camera

0 and 1 in the unburned and burned gas respectively. This binarization procedure leads irremediably to a digitization noise (pixelization) which, in the present case, is smoothed using a low-pass Gaussian filter, with filter size equal to 3 times the spatial resolution. It was checked that doubling filter size did not yield observable changes on the properties of interest (namely the flame wrinkling distribution §III). This indicates that the present measurements are well resolved and that the filter size that is used here is much smaller than that of the smallest flame wrinkling characteristic length-scale. An example of flame contour detection, superimposed on the original image is presented as an illustration in Fig. 2. For the present case, we made the choice of focusing only on the longest contour representing the largest topologically connected object, whereas holes and pockets are not taken into account. The contribution of these missing flame holes and pockets to e.g. the flame surface density was rather limited notwithstanding the relatively low turbulence intensity of our experiments. Future work is however needed to incorporate these disconnected objects into a more self-consistent description. The axis coordinate system is the following, the streamwise distance $x$ coincides with the direction of the bulk flow whereas the transverse distance is noted $y$ (Fig. 2).

A total of 24 different operating conditions have been selected (see Table I). For all cases, the mixture inlet velocity $U_{d}$ is equal to $3.6 \mathrm{~m} \cdot \mathrm{s}^{-1}$ and the mixture of fuel and oxidizer is created well before the burner inlet so that the composition is perfectly premixed. Four pressure magnitudes are investigated from 0.1 to $0.4 \mathrm{MPa}$. Three different fuels are concerned: methane, propane, methane+hydrogen. The laminar flame speed $S_{l}$ and thickness $\delta_{l}=\alpha / S_{l}$ ( $\alpha$ is the fresh gas thermal diffusivity) have been calculated using the Python Cantera library with the GRI-Mech 


\begin{tabular}{|c|c|c|c|c|c|c|c|c|c|c|c|}
\hline Case & Frame rate & Fuel & $\mathrm{P}(\mathrm{bar})$ & $\phi$ & $S_{l}\left(\mathrm{~m} \cdot \mathrm{s}^{-1}\right)$ & $\delta_{l}(\mathrm{~mm})$ & $H_{f}(\mathrm{~mm})$ & $u^{\prime} / S_{l}$ & $L_{t} / \delta_{l}$ & $K a$ & $R e_{t}$ \\
\hline 1 & $10 \mathrm{~Hz}$ & $\mathrm{CH}_{4}$ & 1 & 0.7 & 0.192 & 0.117 & 66.9 & 2.18 & 49.16 & 0.47 & 159 \\
\hline 2 & $10 \mathrm{~Hz}$ & $\mathrm{CH}_{4}$ & 1 & 0.85 & 0.310 & 0.073 & 34.0 & 1.79 & 68.49 & 0.29 & 182 \\
\hline 3 & $10 \mathrm{~Hz}$ & $\mathrm{CH}_{4}$ & 1 & 1.0 & 0.376 & 0.060 & 33.9 & 1.47 & 83.18 & 0.20 & 182 \\
\hline 4 & $10 \mathrm{~Hz}$ & $\mathrm{CH}_{4}$ & 3 & 0.7 & 0.111 & 0.067 & 40.5 & 4.66 & 76.15 & 0.83 & 526 \\
\hline 5 & $10 \mathrm{~Hz}$ & $\mathrm{CH}_{4}$ & 4 & 0.9 & 0.190 & 0.030 & 28.8 & 3.06 & 164.43 & 0.30 & 750 \\
\hline 6 & $10 \mathrm{~Hz}$ & $\mathrm{C}_{3} \mathrm{H}_{8}$ & 1 & 0.8 & 0.363 & 0.062 & 53.2 & 1.28 & 87.67 & 0.16 & 166 \\
\hline 7 & $10 \mathrm{~Hz}$ & $70 \% \mathrm{CH}_{4}+30 \% \mathrm{H}_{2}$ & 1 & 0.6 & 0.114 & 0.198 & 52.6 & 4.66 & 27.32 & 1.65 & 167 \\
\hline 8 & $10 \mathrm{~Hz}$ & $80 \% \mathrm{CH}_{4}+20 \% \mathrm{H}_{2}$ & 1 & 0.7 & 0.210 & 0.107 & 44.8 & 2.39 & 48.76 & 0.54 & 172 \\
\hline 9 & $10 \mathrm{~Hz}$ & $\mathrm{CH}_{4}$ & 1 & 0.8 & 0.269 & 0.084 & 46.5 & 1.83 & 63.00 & 0.31 & 171 \\
\hline 10 & $10 \mathrm{~Hz}$ & $70 \% \mathrm{CH}_{4}+30 \% \mathrm{H}_{2}$ & 2 & 0.6 & 0.093 & 0.120 & 46.3 & 5.31 & 43.71 & 1.64 & 342 \\
\hline 11 & $10 \mathrm{~Hz}$ & $80 \% \mathrm{CH}_{4}+20 \% \mathrm{H}_{2}$ & 2 & 0.7 & 0.151 & 0.074 & 40.7 & 3.44 & 69.21 & 0.67 & 351 \\
\hline 12 & $10 \mathrm{~Hz}$ & $\mathrm{CH}_{4}$ & 2 & 0.8 & 0.191 & 0.059 & 39.4 & 2.76 & 86.92 & 0.43 & 354 \\
\hline 13 & $10 \mathrm{~Hz}$ & $70 \% \mathrm{CH}_{4}+30 \% \mathrm{H}_{2}$ & 3 & 0.6 & 0.059 & 0.127 & 43.1 & 8.63 & 40.80 & 2.87 & 521 \\
\hline 14 & $10 \mathrm{~Hz}$ & $80 \% \mathrm{CH}_{4}+20 \% \mathrm{H}_{2}$ & 3 & 0.7 & 0.121 & 0.062 & 34.3 & 4.58 & 80.39 & 0.79 & 547 \\
\hline 15 & $10 \mathrm{~Hz}$ & $\mathrm{CH}_{4}$ & 3 & 0.8 & 0.165 & 0.045 & 32.3 & 3.45 & 108.44 & 0.44 & 555 \\
\hline 16 & $10 \mathrm{~Hz}$ & $\mathrm{CH}_{4}$ & 2 & 0.77 & 0.190 & 0.059 & 42.2 & 2.67 & 87.95 & 0.41 & 347 \\
\hline 17 & $10 \mathrm{kHz}$ & $\overline{\mathrm{CH}_{4}}$ & 1 & 0.6 & 0.114 & 0.197 & 78.8 & 3.41 & 30.74 & 1.15 & 155 \\
\hline 18 & $10 \mathrm{kHz}$ & $\mathrm{CH}_{4}$ & 1 & 0.7 & 0.192 & 0.117 & 66.3 & 2.19 & 49.02 & 0.47 & 159 \\
\hline 19 & $10 \mathrm{kHz}$ & $\mathrm{CH}_{4}$ & 1 & 0.8 & 0.269 & 0.084 & 48.4 & 1.77 & 63.95 & 0.30 & 168 \\
\hline 20 & $10 \mathrm{kHz}$ & $\mathrm{CH}_{4}$ & 2 & 0.6 & 0.076 & 0.147 & 76.0 & 5.21 & 40.77 & 1.65 & 313 \\
\hline 21 & $10 \mathrm{kHz}$ & $\mathrm{CH}_{4}$ & 3 & 0.6 & 0.059 & 0.127 & 74.9 & 6.80 & 46.81 & 1.87 & 471 \\
\hline 22 & $10 \mathrm{kHz}$ & $\mathrm{CH}_{4}$ & 3 & 0.8 & 0.165 & 0.045 & 34.6 & 3.27 & 110.72 & 0.40 & 539 \\
\hline 23 & $10 \mathrm{kHz}$ & $70 \% \mathrm{CH}_{4}+30 \% \mathrm{H}_{2}$ & 1 & 0.6 & 0.114 & 0.198 & 56.6 & 3.93 & 28.01 & 1.50 & 163 \\
\hline 24 & $10 \mathrm{kHz}$ & $80 \% \mathrm{CH}_{4}+20 \% \mathrm{H}_{2}$ & 1 & 0.7 & 0.210 & 0.107 & 48.4 & 2.27 & 49.91 & 0.49 & 168 \\
\hline
\end{tabular}

TABLE I. Table of operating conditions. The frame rate of the camera is given. The fuel composition, equivalence ratio $\phi$ together with the laminar flame speed $S_{l}$ and thickness $\delta_{l}=\alpha / S_{l}$ are also provided. $H_{f}$ is the flame height, defined as the streamwize distance $x$ at which the mean progress variable $\bar{c}=50 \%$. The indicated values for the turbulent velocity $u^{\prime}$ and integral length scale $L_{t}$ are the average values over the domain $0<x<H_{f}$. The same applies for the Reynolds number $R e_{t}$ and Karlovitz number Ka. As a reminder, case $\{1-18\},\{7-23\},\{8-24\},\{9-19\}$ and $\{15-22\}$ are the same operating conditions investigated using either high or low frame rate tomography.

[56] ver.3.0 mechanism.

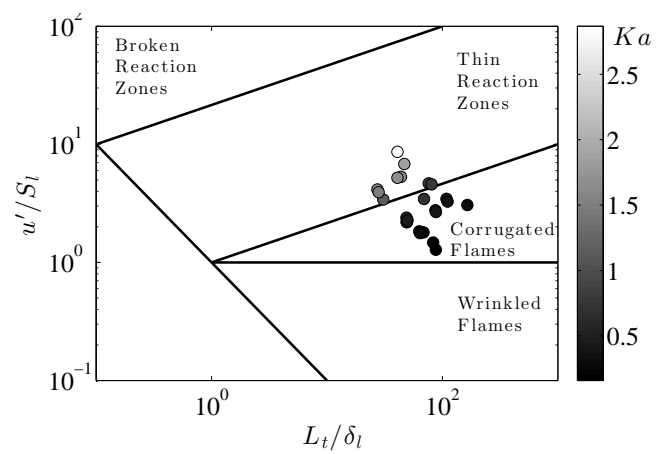

FIG. 3. Experimental conditions overlaid on the premixed combustion regime diagram. The color of the symbols refers to the value of the Karlovitz number

Because of the statistical inhomogeneity of the velocity field in the streamwise direction (transverse inhomogeneity was found to be much smaller), it is rather mistaken to assign a unique value for the turbulence parameters. Therefore, as an illustration, all turbulent quantities detailed in Table I are the average values over a domain extending from the burner inlet to the position $H_{f} \equiv x$ at which the mean progress variable $\bar{c}$ is equal to $50 \%$.

We found that the ratio of the turbulent velocity $u^{\prime}$ to the laminar flame speed $S_{l}$ ranges from about 1.2 to 9 . The ratio of the integral length-scale $L_{t}$ to the laminar flame thickness $\delta_{L}$ extends from about 25 to 160 . The Karlovitz number was calculated as $K a=\tau_{\eta} / \tau_{c}$ where $\tau_{\eta}=(\nu / \epsilon)^{0.5}$ is the Kolmogorov time scale $(\nu$ is the fresh gas viscosity and $\epsilon=15 \nu \overline{(\partial u / \partial x)^{2}}$ is the surrogate of the mean turbulent kinetic energy dissipation rate) and $\tau_{c}=\delta_{l} / S_{l}$ is the chemical time scale. We found that the Karlovitz number is in the range $0.16<K a<2.86$. The Reynolds number $R e_{t}$ based on the integral length-scale $L_{t}$ and the turbulent velocity $u^{\prime}$ extends from 150 to 750 . Note that some operating conditions (Flames $\{1-18\},\{7-23\},\{8-24\}$, $\{9-19\},\{15-22\})$ have been assessed using both low and high frame rate measurements so that comparison between these two techniques can be handled.

The different operating conditions are reported onto the Borghi-Peters diagram $[1,57]$ in Fig. 3. All the operating conditions lie between the corrugated and the thin reaction zone regimes.

\section{B. The POD algorithm}

In the present study, the spatio-temporal features of the flame fronts are assessed by use of POD. For detailed mathematical description of POD and many applications the reader can refer to the review by 
Berkooz et al. [41]. In this subsection, details about the pre- and post-processing steps are depicted.

The spatial coordinates $\left(x_{i, j}\right.$ and $\left.y_{i, j}\right)$ of the flame contour (the black curve in Fig. 2) are independently re-sampled using a fixed number of points $N_{p}$ (hereafter $\left.N_{p}=2000\right) . x_{i, j}$ and $y_{i, j}$ are then rearranged in column vectors and concatenated in a matrix called the snapshot matrix $X_{i, j}$, viz.

$$
X_{i, j}=\left(\begin{array}{ccccc}
x_{1,1} & \cdots & x_{1, j} & \cdots & x_{1, N_{i m}} \\
\vdots & \vdots & \vdots & \vdots & \vdots \\
x_{i, 1} & \cdots & x_{i, j} & \cdots & x_{i, N_{i m}} \\
\vdots & \vdots & \vdots & \vdots & \vdots \\
x_{N_{p}, 1} & \cdots & x_{N_{p}, j} & \cdots & x_{N_{p}, N_{i m}} \\
y_{1,1} & \cdots & y_{1, j} & \cdots & y_{1, N_{i m}} \\
\vdots & \vdots & \vdots & \vdots & \vdots \\
y_{i, 1} & \cdots & y_{i, j} & \cdots & y_{i, N_{i m}} \\
\vdots & \vdots & \vdots & \vdots & \vdots \\
y_{N_{p}, 1} & \cdots & y_{N, j} & \cdots & y_{N_{p}, N_{i m}}
\end{array}\right) .
$$

The subscript $i \in\left[1: N_{p}\right]$ stand for the contour point number and $j \in\left[1: N_{i m}\right]$ is the image number. Consequently, the matrix $X_{i, j}$ is $2 N_{p} \times N_{i m}$. From this snapshot matrix, one can compute some basic statistics such as the mean flame position $\bar{X}_{i}$, the standard deviation $X_{i}^{\prime}$ or the probability of finding the flame at a given position in the flow. We now define the normalized flame position matrix as

$$
\chi_{i, j}=\frac{X_{i, j}-\bar{X}_{i}}{X_{i}^{\prime}}
$$

POD algorithm describes the data using a global approach based on an energetic representation. This means that local features can be either overestimated or underestimated depending on whether or not their associated energy is high or low in the control domain. Even though the turbulent flow corrugating the flame is nearly homogeneous, in this study, the flame front is inhomogeneous since the standard deviation $X_{i}^{\prime}$ (the flame brush) increases monotonically with the streamwise distance $[58,59]$. Therefore, the POD decomposition based on $X_{i, j}$ would have been biased since the first modes (the most energetic) are localized only on the flame tip where $X_{i}^{\prime}$ is maximum but are not present at the base of the flame where $X_{i}^{\prime}$ is smaller. To circumvent this bias, we decided to apply the POD on the reduced flame coordinates $\chi_{i, j}$ since $\chi_{i, j}^{\prime}$ is in essence homogeneous.

The POD algorithm consists in determining a decomposition of the snapshot matrix in a sum of $k$ deterministic functions $\Phi_{k}(\vec{x})$ or $\Phi_{i, k}$ called modes that depend only on space, multiplied by temporal coefficients $\Psi_{k}(t)$ or $\Psi_{k, j}$ weighted by a factor $W_{k}$, viz.

$$
\chi_{i, j}=\sum_{k=1}^{N_{m}} \Phi_{i, k} \times \Psi_{k, j} \times W_{k}
$$
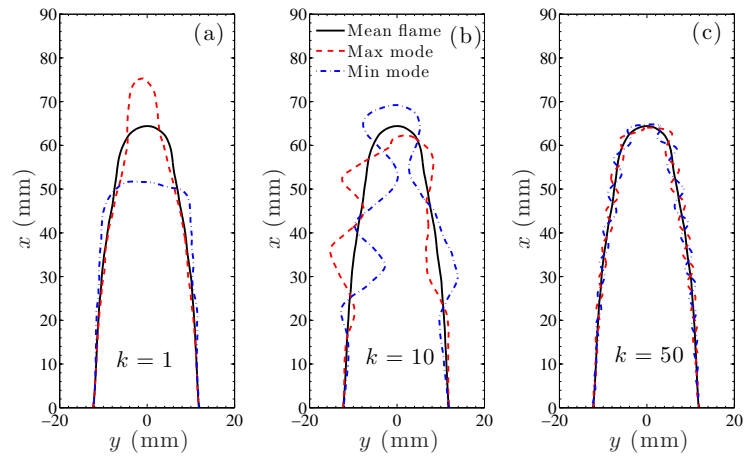

FIG. 4. (Color online). Example of the POD decomposition applied to the flame contours coordinates of Flame 18 (Table I). The mean flame coordinates are represented by the black full line, while the blue dash-dot and red dashed lines correspond respectively to $X_{i, k}^{R}=\bar{X}_{i}+\left(\Phi_{i, k} W_{k} \min \left\{\Psi_{k, j}\right\}\right) X_{i}^{\prime}$ and $X_{i, k}^{R}=\bar{X}_{i}+$ $\left(\Phi_{i, k} W_{k} \max \left\{\Psi_{k, j}\right\}\right) X_{i}^{\prime}$, with $k=1$ (left), $k=10$ (center) and $k=50$ (right).

In Eq. (3), $N_{m}$ is the number of modes used for the reconstruction and is discussed in Appendix A. Eq. (3) can also be rewritten in a matrix formulation as

$$
\chi=\Phi W \Psi .
$$

In the context of POD, the mathematical constraint on the deterministic functions and temporal coefficients is that they are both orthogonal regarding the inner product on the integrable squared function space $\mathcal{L}^{2}$, viz.

$$
\begin{aligned}
& \sum_{j} \Psi_{k, j} \Psi_{l, j}=\delta_{k, l}, \\
& \sum_{j} \Phi_{j, k} \Phi_{j, l}=\delta_{k, l},
\end{aligned}
$$

where $\delta_{k, l}$ is the Kronecker symbol. Eqs. (5a) and (5b) can be recast in matrix formulation as

$$
\begin{gathered}
\boldsymbol{\Psi} \boldsymbol{\Psi}^{T}=\boldsymbol{I}, \\
\boldsymbol{\Phi} \boldsymbol{\Phi}^{T}=\boldsymbol{I},
\end{gathered}
$$

where $\boldsymbol{I}$ is the identity matrix and the superscript $T$ designates the transposed matrix.

By convention the modes and the temporal coefficients are sorted from the largest to the smallest eigenvalue. For this reason, the POD is often referred to as an energy-driven decomposition.

One of the main advantage of using POD is that the flame contours can be reconstructed mode-by-mode. The reconstructed flame coordinates $X_{i, j, k}^{R}$ (where the superscript $R$ stands for 'reconstructed') which thus depend on space $i \in\left[1: N_{p}\right]$, on time (or image number) $j \in\left[1: N_{i m}\right]$ and mode number $k \in\left[1: N_{m}\right]$ are given by

$$
X_{i, j, k}^{R}=\left(\begin{array}{c}
x_{i, j, k}^{R} \\
y_{i, j, k}^{R}
\end{array}\right)=\bar{X}_{i}+\left(\Phi_{i, k} \Psi_{k, j} W_{k}\right) \times X_{i}^{\prime} .
$$




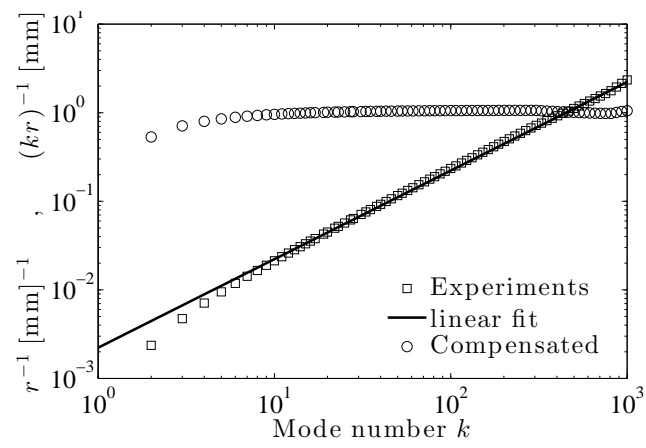

FIG. 5. Correspondance between mode numbers $k$ and wavenumbers $r^{-1}$ for Flame 18 (Table I). A clear linear slope, i.e. $r^{-1} \propto k$ is observed as also confirmed by the compensated wavenumber $(k r)^{-1}$

An example is given in Fig. 4 in which the flame front coordinates are reconstructed using three distinct modes $k=1,10,50$. Specifically, the mean flame coordinates $\bar{X}_{i}$ (the black curves), the reconstructed flame contour of minimum amplitude $X_{i, k}^{R}=\bar{X}_{i}+$ $\left(\Phi_{i, k} W_{k} \min \left\{\Psi_{k, j}\right\}\right) X_{i}^{\prime}$ (green curves) and of maximum amplitude $X_{i, k}^{R}=\bar{X}_{i}+\left(\Phi_{i, k} W_{k} \max \left\{\Psi_{k, j}\right\}\right) X_{i}^{\prime}$ (red curves) are represented in this figure. As expected, the first mode $k=1$ is associated with larger amplitudes by comparison with the mode number 10 and 50. Noticeable is the fact that fluctuations associated with $k=1$ are mostly perceptible at the tip of the flame.

Even though not clearly discernible for $k=1$, one also perceives a hint of periodicity for $k=10$, this periodicity being much more easily distinguishable for $k=50$. This suggests that a given mode number $k$ can be related to a physical spatial scale (wavelength). However, unlike DMD or Fourier transform there is no straightforward relation between POD modes and scales. Here, the following procedure is proposed. (i) the curvilinear coordinate $s$ of the mean flame of coordinates $(\bar{x}, \bar{y})$ is calculated, viz.

$$
s=\int_{\delta_{\tau}}^{N_{p} \delta_{\tau}} \sqrt{\left(\frac{\partial \bar{x}}{\partial \tau}\right)^{2}+\left(\frac{\partial \bar{y}}{\partial \tau}\right)^{2}} d \tau,
$$

where $\tau=\left[1: N_{p}\right] \delta_{\tau}$ is the curvilinear parameter. Then, (ii), the complex number $z_{j, k}^{R}(s)=x_{j, k}^{R}(s)+$ $i y_{j, k}^{R}(s)$ where here $i=\sqrt{-1}$ is calculated and (iii) the Fourier transform of the modulus $\left|z_{j, k}^{R}\right|(s)$ is calculated. $\left|z_{j, k}^{R}\right|(s)$ corresponds to the absolute distance between the reconstructed coordinates of mode $k$ and mean flame positions. (iv) Finally the physical spatial scale $r(k)$ associated with the mode $k$ is identified as the inverse of the wavenumber at which the spectrum of $\left|z_{j, k}^{R}\right|(s)$ is maximum.

Results of this procedure are presented in Fig. 5. Except for mode numbers $k<10$, a clear linear dependence between the mode number and the wavenumber at which the contour spectrum is max- imum is evidenced, i.e $r^{-1}=A_{k r} k$ with $A_{k r}$ the coefficient of proportionality. This is confirmed by the plateau that extends over more than two decades for the compensated wavenumber $\left(A_{k r} k r\right)^{-1}$. Therefore, this procedure allows $A_{k r}$ to be estimated for each operating condition, in order to assign a physical spatial scale to a mode number.

\section{SCALE-BY-SCALE ANALYSIS OF THE FLAME WRINKLING}

\section{A. Definition, Parametrization and comparison with the caliper technique}

We now turn our attention to the contribution of each scale to the flame wrinkling. Since only 2D images of the flame contours are accessible in the present study, our focus will be on the contribution of each scale to the arclength of the interface separating the fresh to the burned gas. This provides a measure of the degree of wrinkling (or roughness) at a given scale (see for instance $[32,60]$ and references therein). In this goal, the POD algorithm is first employed to reconstruct the flame coordinates $\left(x_{i, j, 1: k}^{R}, y_{i, j, 1: k}^{R}\right)$ from the first mode up to a given mode $k$, viz.

$$
\left(\begin{array}{c}
x_{i, j, 1: k}^{R} \\
y_{i, j, 1: k}^{R}
\end{array}\right)=\bar{X}_{i}+X_{i}^{\prime} \sum_{m=1}^{k}\left(\Phi_{i, m} \Psi_{m, j} W_{m}\right) .
$$

This is equivalent of low-pass filtering the flame front coordinates. Then, the scale-by-scale contribution to the arclength of the flame front is defined as

$$
L(k)=\frac{1}{N_{i m}} \sum_{j=1}^{j=N_{i m}} \int_{\delta_{\tau}}^{N_{p} \delta_{\tau}} s(\tau) d \tau
$$

where

$$
s(\tau)=\left[\left(\frac{\partial x_{\tau, j, 1: k}^{R}}{\partial \tau}\right)^{2}+\left(\frac{\partial y_{\tau, j, 1: k}^{R}}{\partial \tau}\right)^{2}\right]^{1 / 2}
$$

is the curvilinear coordinates along the flame contours reconstructed using $k$ modes. Recalling that the scale $r$ is related to the mode number $k$ via the relation $r=\left(A_{k r} k\right)^{-1}$, the arclength $L(k)$ can be plotted also as a function of the physical scale $r$. Notwithstanding the analogy between POD reconstructions and classical filtering, this approach turns out to be rather similar to that employed by [32] where the coarsegraining of the interface was done using a box filter of varying filter size. An example is given in Fig. 6 where the flame arclength $L(r)$ is normalized by the arclength of the (mean) non wrinkled interface, hereafter noted $L_{0}$. The shape of $L(r)$ is quite similar to that observed in many previous studies (see for instance $[32,60])$. At large scales, $L(r) / L_{0}$ tends to unity, then increases rapidly as we travel through 


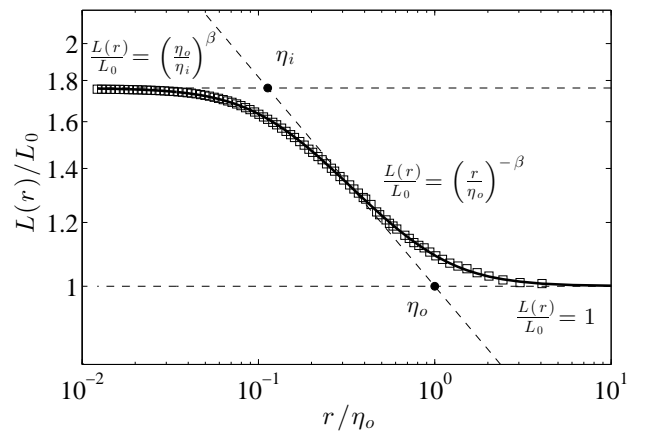

FIG. 6. Assessment of the curvilinear arclength $L / L_{0}$ of the flame contour as a function of the scale $r / \eta_{o}$ through the POD technique (symbols) for Flame 1. Also plotted is the parametric expression Eq. (12) (full line) where the parameters $\eta_{o}, \eta_{i}$ and $\beta$ have been inferred from non linear least square fit.

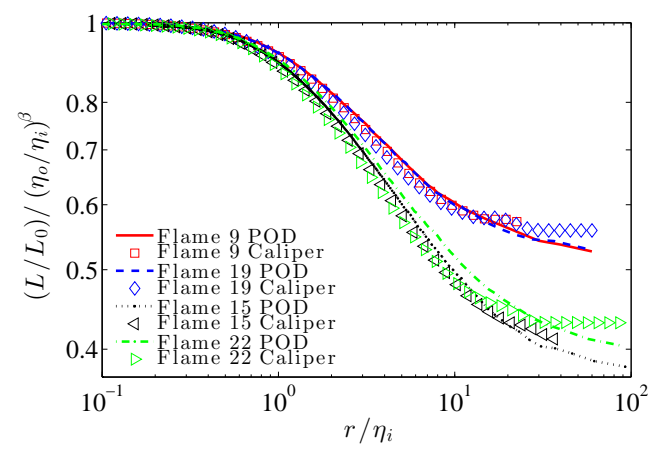

FIG. 7. (Color online). Comparison of the POD (lines) and caliper (symbols) techniques for assessing $L / L_{0}$ for case $9,19,15$ and 22 . The arclength is normalized by the small-scale quantities, i.e. $L / L_{0}\left(\eta_{o} / \eta_{i}\right)^{-\beta}$ vs $r / \eta_{i}$

scales of smaller size before reaching a plateau at very small scales. This curve, known as the Richardson plot in fractal analysis, allows the main characteristics of the flame wrinkling to be identified as described below.

It is well known $[29,30]$ that the fractal facet of folded interfaces is observed over a range of scales spanning from an outer cut-off (a large scale) $\eta_{o}$ to an inner cut-off (a small scale) $\eta_{i}$. Within this range, it is observed that the arclength of a wrinkled interface follow a power-law evolution of the form $L(r) \propto r^{-\beta}$ where $\beta$ is related to the so-called fractal dimension (Fig. 6). For scales much larger than $\eta_{o}, L(r)$ is constant and is equal to $L_{0}$ (Fig. 6). When the scale $\eta_{i} \gg r$, we have $L(r) / L_{0}=\left(\eta_{o} / \eta_{i}\right)^{\beta}[29,30]$. These three distinct scaling can be matched together in a single parametric expression of the form

$$
\frac{L(r)}{L_{0}}=\left(\frac{\eta_{o}}{\eta_{i}}\right)^{\beta}\left[\frac{1+\left(\frac{r}{\eta_{o}}\right)^{2}}{1+\left(\frac{r}{\eta_{i}}\right)^{2}}\right]^{\beta / 2} .
$$

This matched-scaling function is very similar to that first initiated by Batchelor [61] and further extensively used in e.g. [62-67] for describing velocity or scalar structure functions $[68,69]$ in turbulent flows, where three distinct scaling at respectively large, small and intermediate scales are also observed.

In the context of fractal analysis, after extensively scrutinizing the literature, it was found that a similar equation to Eq. (12) was first suggested by Mandelbrot for describing the 'rank-frequency' relation of words in literature [70]. This relation is sometimes referred to as the Zipf-Mandelbrot law [71, 72]. It was further employed by Rigaut $[71,72]$ for describing the coverage length of pulmonary alveolus. In $[71,72]$, this expression was used to justify the systematic deviations from a pure fractal behaviour at very small value of $r$. He called this type of behaviour "semi-fractal' since the fractal dimension appeared to be scale-dependent. Our claim is here very different since the bending of fractal plots at small and large scales is in our case related to the inner and outer cut-off but not to a change in fractal dimension which remains constant independent of $r$.

The appropriateness of Eq. (12) to describe the scale-by-scale contribution to the flame wrinkling is illustrated in Fig. 6. The parameters $\eta_{o}, \eta_{i}$ and $\beta$ have been here inferred from a non linear least mean square fit using the function nlinfit of MATLAB. The normalized standard deviation between experiments and the fitting function is always of the order $10^{-4}$ which demonstrates a posteriori the perfect adequacy of Eq. (12) for characterizing $L(r)$. The main advantage of using this parametric expression is that the assessment of the three parameters $\eta_{o}, \eta_{i}$ and $\beta$ is totally unambiguous and do not imply any degree of arbitrariness notably in the estimation of the 'best' range of scaling in the inertial range which spans between $\eta_{i}<r<\eta_{o}$. This is even more relevant for low to moderate Reynolds numbers.

In the goal of validating the present assessment of the scale-by-scale properties of the turbulent flame fronts, we carried out a comparison with the caliper technique. In the caliper technique [73], the length of a curve is estimated by counting the number of ruler lengths of size $r$ to cover the whole length of the curve. This process is repeated for a set of ruler lengths. In the present case, use was made of the algorithm proposed in [60, 74].

Results are presented in Fig. 7 where the scale-byscale contribution to the arclength as obtained using either the POD or the caliper technique is shown. Two couples of operating conditions, i.e. Flames (919) and Flames (15-22) are considered to compare the assessments made using either the high or the low frame rate tomography technique.

POD analysis is based on a statistical approach [41]. Furthermore, since the decomposition is datadriven, it may be sensitive to convergence and statistical independence of flow realizations. These two constraints may lead to severe issues as far as high- 


\begin{tabular}{|c|c|c|c|c|c|c|c|c|}
\hline \multirow[b]{2}{*}{ Case } & \multicolumn{4}{|c|}{ POD } & \multicolumn{4}{|c|}{ caliper } \\
\hline & $\eta_{o}[\mathrm{~mm}]$ & $\eta_{i}[\mathrm{~mm}]$ & $\beta$ & $\left(\frac{\eta_{o}}{\eta_{i}}\right)^{\beta}$ & $\eta_{o}[\mathrm{~mm}]$ & $\eta_{i}[\mathrm{~mm}]$ & $\beta$ & $\left(\frac{\eta_{o}}{\eta_{i}}\right)^{\beta}$ \\
\hline 1 & 16.5 & 1.87 & 0.26 & 1.76 & 12.1 & 2.24 & 0.31 & 1.70 \\
\hline 2 & 11.8 & 1.11 & 0.29 & 1.98 & 13.8 & 1.61 & 0.29 & 1.86 \\
\hline 3 & 15.3 & 1.41 & 0.24 & 1.78 & 13.4 & 1.69 & 0.26 & 1.71 \\
\hline 4 & 11.1 & 0.68 & 0.32 & 2.45 & 13.1 & 1.33 & 0.36 & 2.29 \\
\hline 5 & 19.6 & 0.72 & 0.30 & 2.71 & 19.4 & 1.06 & 0.30 & 2.38 \\
\hline 6 & 15.2 & 1.31 & 0.25 & 1.84 & 12.6 & 1.59 & 0.27 & 1.76 \\
\hline 7 & 13.7 & 1.17 & 0.29 & 2.05 & 16.9 & 1.64 & 0.30 & 2.03 \\
\hline 8 & 14.8 & 1.04 & 0.27 & 2.00 & 16.4 & 1.48 & 0.28 & 1.96 \\
\hline 9 & 11.6 & 1.22 & 0.25 & 1.86 & 17.5 & 1.47 & 0.25 & 1.86 \\
\hline 10 & 12.4 & 0.69 & 0.34 & 2.61 & 16.5 & 1.22 & 0.36 & 2.59 \\
\hline 11 & 13.7 & 0.77 & 0.33 & 2.51 & 15.7 & 1.21 & 0.35 & 2.48 \\
\hline 12 & 12.0 & 0.82 & 0.31 & 2.37 & 15.8 & 1.21 & 0.33 & 2.33 \\
\hline 13 & 12.0 & 0.61 & 0.34 & 2.88 & 14.7 & 1.09 & 0.40 & 2.85 \\
\hline 14 & 12.9 & 0.56 & 0.33 & 2.75 & 15.1 & 0.97 & 0.36 & 2.71 \\
\hline 15 & 12.2 & 0.62 & 0.32 & 2.62 & 15.1 & 0.94 & 0.33 & 2.54 \\
\hline 16 & 12.2 & 0.92 & 0.29 & 2.13 & 12.1 & 1.55 & 0.35 & 2.06 \\
\hline 17 & 21.1 & 1.73 & 0.20 & 1.66 & 16.2 & 1.61 & 0.23 & 1.71 \\
\hline 18 & 22.0 & 1.72 & 0.25 & 1.87 & 15.6 & 1.97 & 0.29 & 1.83 \\
\hline 19 & 23.9 & 1.87 & 0.25 & 1.87 & 14.6 & 1.78 & 0.28 & 1.81 \\
\hline 20 & 17.6 & 1.06 & 0.27 & 2.15 & 15.0 & 1.69 & 0.35 & 2.16 \\
\hline 21 & 15.4 & 0.79 & 0.29 & 2.40 & 15.6 & 1.52 & 0.38 & 2.42 \\
\hline 22 & 20.8 & 1.03 & 0.30 & 2.45 & 13.2 & 1.26 & 0.36 & 2.34 \\
\hline 23 & 20.9 & 1.55 & 0.27 & 2.04 & 15.2 & 1.89 & 0.33 & 1.99 \\
\hline 24 & 23.2 & 1.61 & 0.25 & 1.95 & 15.5 & 1.69 & 0.28 & 1.87 \\
\hline Error (\%) & & & & & 23.6 & 27.6 & 10.6 & 3.1 \\
\hline
\end{tabular}

TABLE II. (Color online). Parameters $\eta_{o}, \eta_{i}$ and $\beta$ as given by a least square fit using Eq. (12) for all experimental conditions investigated here. The error is computed as the average of $100 \times\left|Q_{\mathrm{POD}} / Q_{\mathrm{Caliper}}-1\right|$ over the 24 conditions, where $Q$ denote the quantity under consideration. Again, case $\{1-18\},\{7-23\},\{8-24\},\{9-19\}$ and $\{15-22\}$ are the same operating conditions investigated using either high or low frame rate tomography.

speed measurements are concerned. Indeed, while using high-speed tomography enables the flame front dynamics to be tracked, two main issues arise: (i) successive snapshots are, by definition, statistically dependent and (ii) the time of measurement is short (camera memory limitation). The conjunction of these two shortcomings may lead in unconverged statistics. By comparing low and high frame rates, we have addressed these issues. According to the POD analysis of our results, we do not see significant differences between both frame rates meaning that statistical convergence has been achieved. Indeed, the agreement between the high and low frame rate tomography is particularly satisfactory, with differences that do not exceed $10 \%$.

Fig. 7 also emphasizes that the POD technique agrees nicely with the caliper method, although some slight discrepancies are observed. This differences is mainly attributed to a well known bias in the caliper technique associated to the fact that the analysed curve do not contain an integer number of rulers $[74,75]$. This effect is mostly visible at large-scales as highlighted in Fig. 7 where it is observed that $L(r)$ issued from either the POD or the caliper technique closely match until a physical scale $r<20 \eta_{i} \approx \eta_{o}$.

A comparison between the POD and caliper methods is further provided in Table II for the 24 differ- ent operating conditions. Some differences are again noticeable. Discrepancies are observed mainly at the level $\eta_{o}$ and $\eta_{i}$ with differences of about $25 \%$, whereas the fractal dimension assessed by the two techniques is within $10 \%$. These differences are again attributed to the bias in the estimation of $L(r)$ at large scales using the caliper technique. This bias affects the fitting procedure for estimating $\eta_{o}$ and so forth $\eta_{i}$. Differences can be also due to the method used to relate a mode number to a physical scale. However, the quantity $\left(\eta_{o} / \eta_{i}\right)^{\beta}$, representing the ratio of the wrinkled to unwrinkled arclength agrees very nicely, independently of the method employed $(\sim 3 \%)$.

In summary, the fractal characteristics of the flame are computed by an unambiguous least-square fit method using the parametric expression Eq. (12). The caliper technique compares favourably well with POD except at large scales for which the caliper technique is known to provide some biased results. The quantity $\left(\eta_{o} / \eta_{i}\right)^{\beta}$ assessed by the two methods is in excellent agreement. Consequently, we conclude that the POD algorithm is reliable for assessing the scaleby-scale contribution to the arclength. 


\section{B. Scale similarity of the flame wrinkling}

In the context of turbulent non-reacting flows, there has been a long-standing quest for assessing the relevant characteristic scales that ought to be invoked for normalizing velocity or scalar energy spectra or structure functions (see e.g. [14, 76] and references therein). For instance, if one observes a collapse of energy spectra over a given range of scales, once normalized by the relevant velocity and length scales, it is stated that these spectra comply with scale similarity over this particular range of scales. If so, scale similarity suggests that energy spectra can be represented by an universal parametric expression [14]. Seeking for such scaling is thus of tremendous importance in the analytical description of turbulence.

In the present study, the same question is addressed for the scale-by-scale contribution to the arclength $L(r)$. Specifically, we investigate the degree with which $L(r)$ complies with scale similarity using different characteristic quantities. In the present case, one can invoke two characteristic length-scales for normalizing $r$, i.e. $\eta_{o}$ and $\eta_{i}$, and two characteristics length-scales for normalizing $L(r)$, namely $L_{0}$ and $L_{0}\left(\eta_{o} / \eta_{i}\right)^{\beta}$. Therefore, one can construct different types of scale similarity, viz.

(i) Large scale similarity, viz.

$$
\frac{L}{L_{0}} \quad \text { vs } \quad \frac{r}{\eta_{o}}
$$

which is equivalent to normalizing velocity energy spectra by the integral length scale and the velocity variance. In this case, scale similarity is expected to hold at large scales.

(ii) Small scale similarity, viz.

$$
\frac{L / L_{0}}{\left(\eta_{o} / \eta_{i}\right)^{\beta}} \text { vs } \frac{r}{\eta_{i}}
$$

which is equivalent to normalizing velocity energy spectra by the Kolmogorov velocity and length scales. In this case, scale similarity is expected to hold at small scales. Note that the small-scale similarity variables do not depend only on some small scales characteristics but depend also on the large-scales through the appearance of $L_{0}$ and $\eta_{o}$.

(iii) Log-similarity. This type of similarity solution originates from non-reacting turbulent flows. It has been first observed and justified for the temperature field in a Rayleigh-Bénard convection flow [77] and further investigated in detail for the velocity field in fully developed homogeneous isotropic turbulence [76, 78-80]. In the context of fluid turbulence, log-similarity is predicted by both the multifractal model and the variational approach of the small scale intermittency $[79,80]$. The first attempt in investigating the appropriateness of log-similarity for fractal plots originates from Meakin [81-83]. When applied to $L(r)$, log-similarity consists in calculating

$$
\mathcal{L}=\frac{\log \left(\frac{L}{L_{0}}\right)}{\beta \log \left(\frac{\eta_{o}}{\eta_{i}}\right)} \quad \text { vs } \mathcal{R}=\frac{\log \left(\frac{r}{\eta_{o}}\right)}{\log \left(\frac{\eta_{o}}{\eta_{i}}\right)} .
$$

$\mathcal{L}$ might be interpreted here as a normalized scale entropy as in $[34,35,84]$. One can further define the scale entropy flux $\mathcal{F}[34,35,84]$

$$
\mathcal{F}=-\frac{\partial \mathcal{L}}{\partial \mathcal{R}} .
$$

$\beta \times \mathcal{F}$ is a measure of the local fractal dimension. Indeed, if $L(r) / L_{0}=\left(r / \eta_{o}\right)^{-\beta}$ in the range $\eta_{i}<$ $r<\eta_{0}$, then it can be readily shown that $\beta \mathcal{F}=$ $-\beta \partial \mathcal{L} / \partial \mathcal{R}=\beta$ over $-1<\mathcal{R}<0$. By injecting Eq. (12) into Eq. (15), it is readily shown that

$$
\mathcal{L}=1+\frac{1}{2 a} \log \left[\frac{1+\left(\frac{r}{\eta_{o}}\right)^{2}}{1+\left(\frac{r}{\eta_{i}}\right)^{2}}\right],
$$

where $a=\log \left(\eta_{o} / \eta_{i}\right)$, i.e. corresponds to the logarithmic separation between $\eta_{o}$ and $\eta_{i}$. At this stage it is easy to notice that $\mathcal{L}$ does not depend on $\beta$. By further using the trigonometric relation $\cosh (x)=\left(e^{2 x}+1\right) / 2 e^{x}$, it can be shown that the scale entropy writes

$$
\mathcal{L}(\mathcal{R})=\frac{1}{2 a}\left\{a+\log \left[\frac{\cosh (a \mathcal{R})}{\cosh (a \mathcal{R}+a)}\right]\right\} .
$$

By differentiating with respect to $\mathcal{R}$, the scale entropy flux reads

$$
\mathcal{F}(\mathcal{R})=-\frac{1}{2}\{\tanh (a \mathcal{R})-\tanh (a \mathcal{R}+a)\} .
$$

Results for the 24 different operating conditions using the three aforementioned sets of similarity variables are presented in Figs. 8 to 11. As one could have expected, none of the scalings based on either outer (large) scales or inner (small) scales lead to a complete collapse over the entire range of scales. Specifically, a strong scatter is observed within the inertial range (if it may be defined as the scale separation between $\eta_{o}$ and $\eta_{i}$ is moderate) where the scaling exponent $\beta$ and $\left(\eta_{o} / \eta_{i}\right)^{\beta}$ are interdependent. This was already noticed by [21] who found that the fractal dimension followed a similar evolution than the flame surface density.

We now turn our attention to the degree with which log-similarity holds for $L(r)$. Figs. 10 and 11 present respectively the scale entropy $\mathcal{L}$ and the scale entropy 


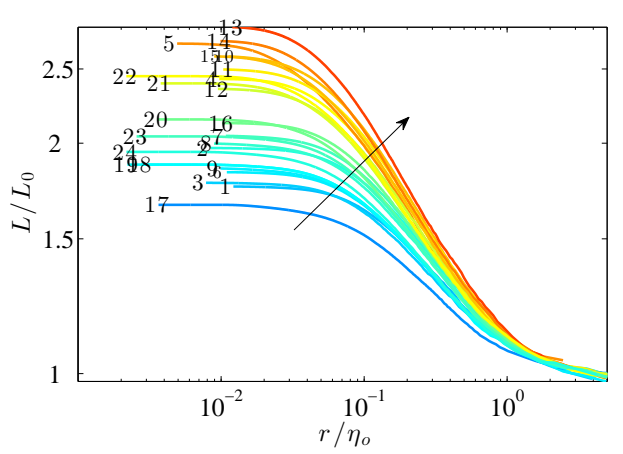

FIG. 8. (Color online). Large-scale similarity, i.e. $L / L_{0}$ is plotted as a function of $r / \eta_{o}$. The arrow indicates increasing values of $\left(\eta_{o} / \eta_{i}\right)^{\beta}$.

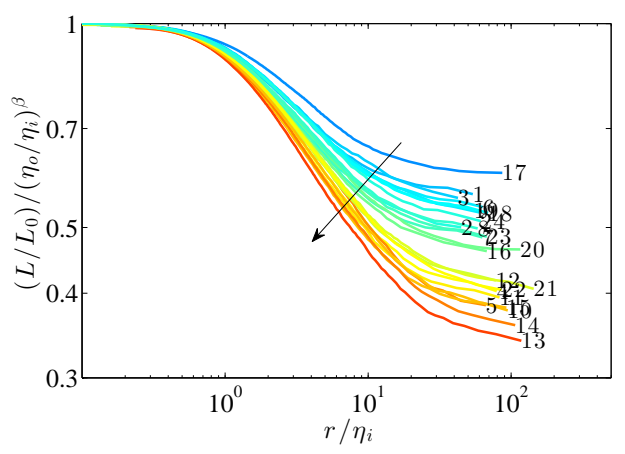

FIG. 9. (Color online). Small-scale similarity, i.e. $L / L_{0}\left(\eta_{o} / \eta_{i}\right)^{-\beta}$ is plotted as a function of $r / \eta_{i}$.

flux $\mathcal{F}$ as a function of the normalized logarithmic separation $\mathcal{R}$. All the curves collapse reasonably well except for a separation $\mathcal{R}$ close to either -1 or 0 corresponding to $r=\eta_{i}$ and $\eta_{o}$ respectively. Note also that the scale entropy distribution differs significantly from that corresponding to an infinite value of $a$, i.e. for an infinite separation between $\eta_{o}$ and $\eta_{i}$.

This departure is more easily discernible when the scale entropy flux is plotted (Fig. 11). Let us recall that $\mathcal{F}$ is a measure of the local (as a function of $\mathcal{R}$ ) fractal dimension. As mentioned above, for a proper inertial range to be defined, the scale entropy flux should reach a value of 1 for scales in the range $-1<\mathcal{R}<0$. Clearly, $\mathcal{F}$ does not reveal any plateau and its maximum value is always below 1 . This might be wrongly interpreted as an evidence of a 'scale-dependent' fractal behaviour as in [31, 71, 72] (sometimes referred to as semi-fractal or multi-fractal behaviour). Although this scenario is physically tenable, the fractal dimension $\beta$ appearing in Eq. (12) is in essence scale-independent. Therefore, this lack of scaling is rather due to the overlapping effect associated with the bending of $L(r)$ near the outer and inner cut-off. By comparing experimental curves to that corresponding to an infinite value of $a$ (black dash-dotted line), it appears that a plateau can indeed be observed but for much higher value of $\eta_{o} / \eta_{i}$.

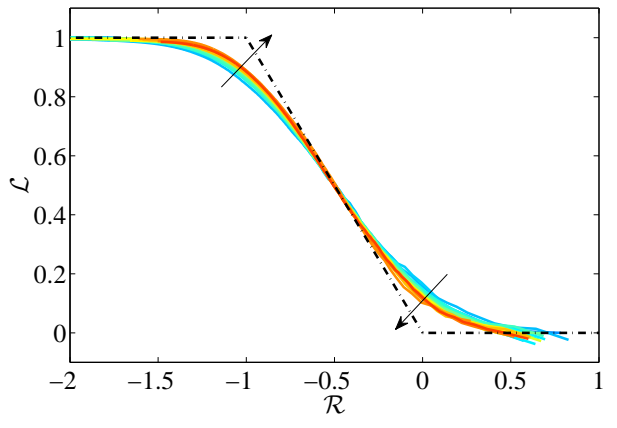

FIG. 10. (Color online). (a) Scale entropy $\mathcal{L}(\mathcal{R})$ as defined by Eq. (15). The black dash-dot lines represents the asymptotic distribution, i.e. $a \rightarrow \infty$.

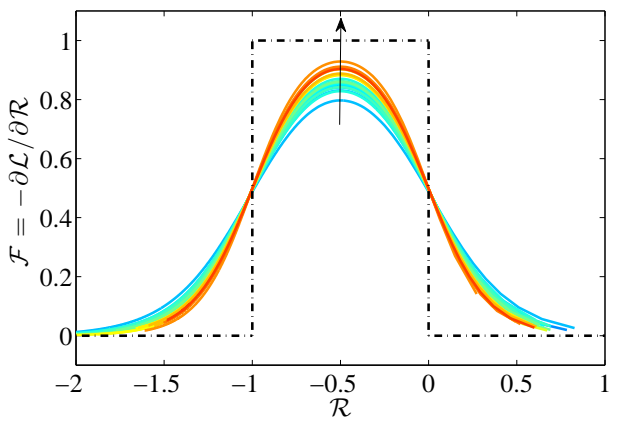

FIG. 11. (Color online). Scale entropy flux $\mathcal{F}(\mathcal{R})$ as given by Eq. (16). The black dash-dotted lines represents the asymptotic distribution, i.e. $a \rightarrow \infty$.

On the basis of Eq. (19), it is possible to assess the conditions needed for $\mathcal{F}_{\max } \equiv \max (\mathcal{F})$ to reveal a plateau. In effect, it is readily shown that

$$
\begin{aligned}
\mathcal{F}_{\max }=\tanh \left(\frac{a}{2}\right) & =1-\frac{2}{\frac{\eta_{o}}{\eta_{i}}+1} \\
& \approx 1-2\left(\frac{\eta_{o}}{\eta_{i}}\right)^{-1},
\end{aligned}
$$

when $\frac{\eta_{o}}{\eta_{i}}$ is sufficiently large.

Fig. 12 depicts the evolution of $\mathcal{F}_{\text {max }}$ as given by Eq. (20) together with the experimental assessments inferred from the present database. This figure reveals that $\mathcal{F}_{\text {max }} \rightarrow 1$ is only approached asymptotically. A value of $\eta_{o} / \eta_{i}$ of the order of $10^{3}$ is needed for $\mathcal{F}_{\max }$ to be equal to one with a reasonable degree of precision. It is worth noticing that the present experimental database for which $10 \lesssim \eta_{o} / \eta_{i} \lesssim 30$ is far from fulfilling this condition. This lack of scaling range is further confirmed when the compensated arclength $L(r) / L_{0}\left(r / \eta_{o}\right)^{\beta}$ (given by Eq. (12)) is plotted as a function of $r / \eta_{o}$ for different ratios $\eta_{o} / \eta_{i}$ (Fig. 13). When $\eta_{o} / \eta_{i}=2.10^{1}$ which is at the midspan of our experiments, the scaling range is very limited and resembles an inflection point rather than a clear plateau. Beyond $1.10^{3}$, one can unambiguously discern a scaling range over about two decades. 


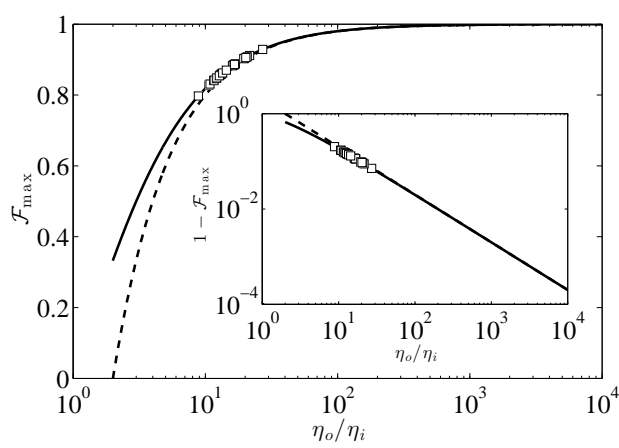

FIG. 12. (Color online). $\mathcal{F}_{\max }$ as a function of the scale ratio $\eta_{o} / \eta_{i}$. The full line represents $\mathcal{F}_{\max }=\tanh \left(\frac{a}{2}\right)$ whilst the dashed line is given by the approximation $1-2\left(\eta_{o} / \eta_{i}\right)^{-1}$, symbols correspond to $\mathcal{F}_{\max }$ for the 24 operating conditions investigated here. The inset shows $1-\mathcal{F}_{\max }$.

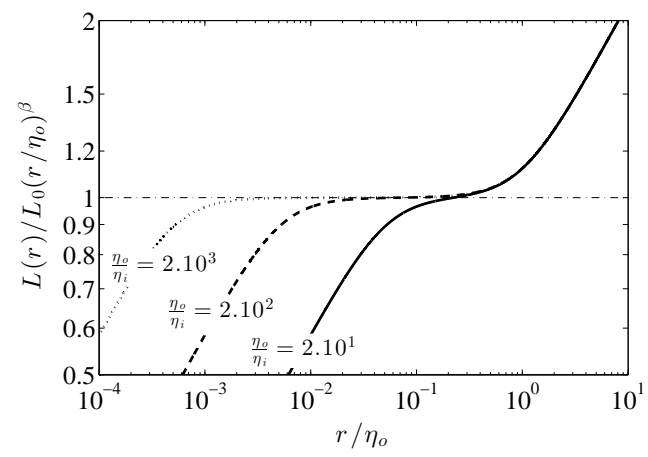

FIG. 13. Compensated arclength $L(r) / L_{0}\left(r / \eta_{o}\right)^{\beta}$ as given by Eq. (12) with $\beta=1 / 3$, for three different values of $\eta_{o} / \eta_{i}=2.10^{1}, 2.10^{2}$ and $2.10^{3}$ for which $1-\mathcal{F}_{\max }$ is respectively $10^{-1}, 10^{-2}$ and $10^{-3}$.

This clearly demonstrates that the finite ratio between $\eta_{o}$ and $\eta_{i}$ has a drastic impact on the distribution of $L(r)$. A finite separation between $\eta_{o}$ and $\eta_{i}$ implies a crossover between the statistical behaviour of large $\left(\equiv \eta_{o}\right)$ and small scales $\left(\equiv \eta_{i}\right)$ which affects the maximum value of the scale entropy flux, i.e. the apparent slope of $L(r)$ in the inertial range. In the field of non reacting turbulent flows, this effect is generally referred to as a finite Reynolds number effect (see for instance $[65,66,85,86]$ and references therein). In this context, the inertial range might be preferably called the restricted scaling range as in e.g. [86].

Therefore, because of the aforementioned finite ratio effect, it is not surprising to observe that scale similarity does not hold in the present case (Figs. 8 and 9). Indeed, one may need to reach much higher values of $\eta_{o} / \eta_{i}$ for large and small scales to behave independently and for an asymptotic value for $\beta$ to be possibly attained. Consequently, finite Reynolds number effects are likely to shed doubts on most of the experimental or numerical estimations of $\beta$ and the quest for an universal value or universal evolution for this parameter is worth being revisited.

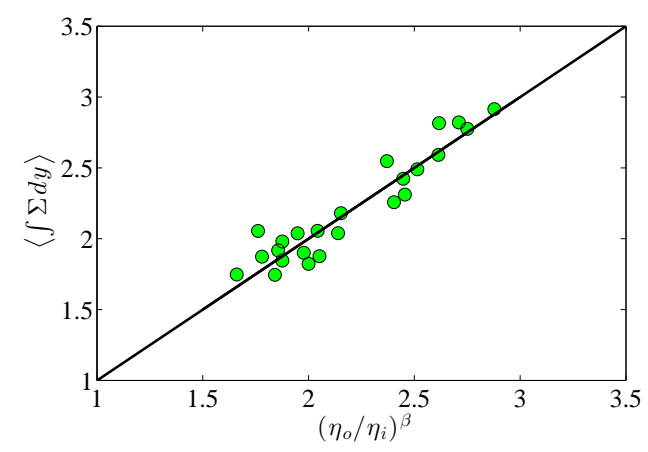

FIG. 14. (Color online). Correlation between the averaged flame surface density and $\left(\eta_{o} / \eta_{i}\right)^{\beta}$.

\section{IMPLICATION FOR MODELLING}

Many studies $[9,11,20]$ have emphasized the ability of fractal models for predicting the flame surface density in either RANS (e.g. $[15,16]$ ) or LES (e.g. $[10,11])$ context. Fig. 14 put emphasis on the excellent correlation between $\left(\eta_{o} / \eta_{i}\right)^{\beta}$ and the averaged flame surface density over the domain. The latter is defined as

$$
\left\langle\int \Sigma d y\right\rangle=\frac{2}{H_{f}} \int_{x=0}^{H_{f}} \int_{y=0}^{\infty} \Sigma(x, y) d x d y,
$$

where the flame surface density $\Sigma(x, y)$ has been estimated using the same procedure as in $[20,87]$. Therefore, the challenge is focused on the predictions of the parameters $\eta_{o}, \eta_{i}$ and $\beta$. We thus now turn our attention to the ability of some available empirical or phenomenological relations for predicting the evolution of the fractal parameters with respect to the flow/flame characteristics.

\section{A. Prediction of the inner and outer cutoffs}

There is wealth of experimental $[17,88]$ data and phenomenological arguments $[11,20,89]$ which suggest that, in analogy with non reacting flows, the inner cut-off is proportional to the Kolmogorov lengthscale. In this case

$$
\frac{\eta_{i}}{\delta_{l}}=C K a^{-1 / 2} .
$$

Using the present definition for $\delta_{l}=\alpha / S_{l}$, Gülder and Smallwood [17] advocated a value for $C$ of 15.4 whilst Roberts et al. [88] found a value of 4.9. Kobayashi et al. [89] suggested that the inner cut-off could also be identified with the cross-over length scale in the kinetic energy spectrum between inertial and dissipative scales. This was further recently confirmed by some physical reasoning arguments [20]. In this case, the value for $C$ relates to the Kolmogorov constant $C_{u}=2$ through $C=r_{1}^{*}=\left(11 C_{u}\right)^{3 / 4} \approx 10.2$. However, this scaling might hold only for sufficiently 


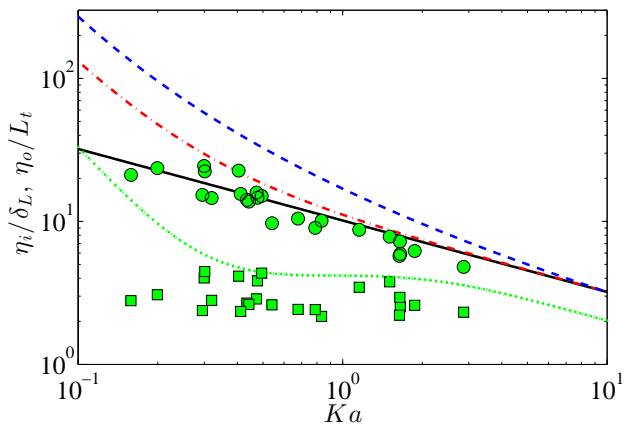

FIG. 15. (Color online). Evolution of the inner (circles) and outer (squares) cutoffs as a function of the Karlovitz number. The inner and outer cutoffs are normalized respectively by the laminar flame thickness $\delta_{l}$ and integral length scale $L_{t}$. The black full line corresponds to Eq. (22) with $C=r_{1}^{*} \approx 10.2$, the red dash-dotted curve is given by Eq. (24), the green dotted curve corresponds to Eq. (25) and the blue dashed line represents Eq. (26)

large values of Karlovitz number so that the flame can be treated as a passive interface. For low Karlovitz numbers, it has been proposed that the inner cutoff should rather scale with the Gibson length-scale $L_{G} / L_{t}=\left(S_{l} / u^{\prime}\right)^{3}[1,11,20]$, which in terms of $\delta_{l}$ writes

$$
\frac{\eta_{i}}{\delta_{l}}=K a^{-2}
$$

To be consistent with both the high and low Karlovitz regimes, Refs. [11, 20] suggested the following expression for $\eta_{i}$

$$
\frac{\eta_{i}}{\delta_{l}}=r_{1}^{*} K a^{-1 / 2}+K a^{-2} .
$$

Using similar arguments, Ref. [90] proposed the following parametrization for $\eta_{i} / \delta_{l}$

$$
\begin{aligned}
\frac{\eta_{i}}{\delta_{l}}= & 0.345 K a^{-2} \exp (-K a) \\
& +6.41 K a^{-1 / 2}[1-\exp (K a)] .
\end{aligned}
$$

Shim et al. [91] have also conjectured that, in analogy with non reacting flows, the inner cut-off should approach the diameter of the most probable vortices $\approx 8 \eta$, viz. [91]

$$
\frac{\eta_{i}}{\delta_{l}}=8 K a^{-1 / 2} \exp \left(\frac{3}{4} K a^{-1 / 2}\right) .
$$

Eqs. (22), (24), (25) and (26) are compared to the present assessments of $\eta_{i}$ in Fig. 15. Although experimental data are relatively scattered, our study confirms the decreasing tendency of $\eta_{i} / \delta_{l}$ with respect to the Karlovitz number as given by Eq. (22) with a constant $C$ consistent with that predicted by [20], i.e. $C=r_{1}^{*} \approx 10.2$. In contrast, the predictions given by Eq. (24) [11, 20], Eq. (25) [90] and Eq. (26) [91] appear to differ significantly from experimental data. Note that care should be paid in extrapolating these

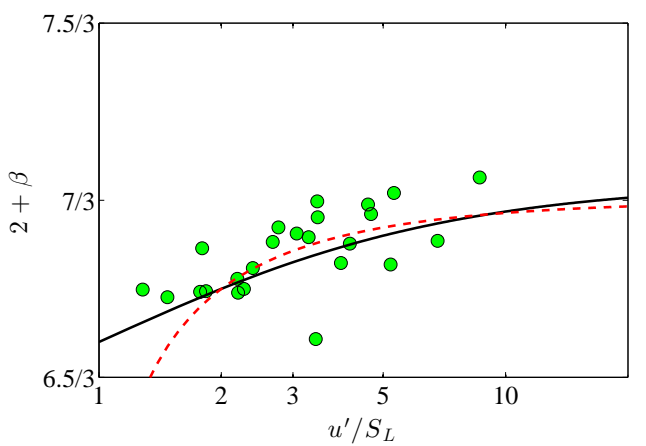

FIG. 16. (Color online). Fractal dimension $\beta$ as a function of $u^{\prime} / S_{l}$. The black full line and red dashed line correspond to Eq. (28) and (27) respectively.

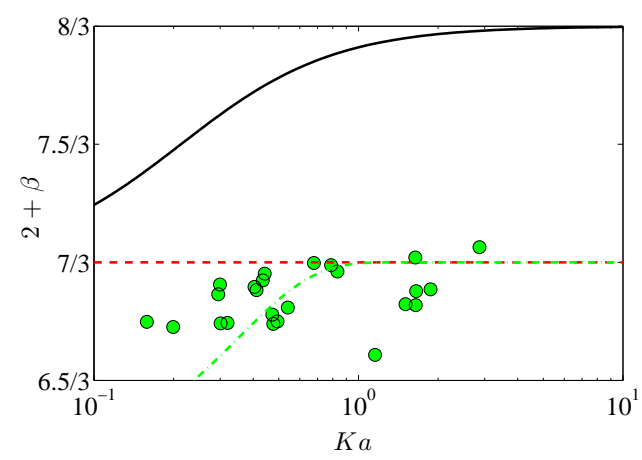

FIG. 17. (Color online). Fractal dimension as a function of $K a$, the red dashed line represents $\beta=1 / 3$, the black curve designates Eq. (30) whilst the green dash-dot line is given by Eq. (29).

conclusions to Karlovitz number beyond the range studied here. Also plotted in Fig. 15 is the ratio between the outer cut-off $\eta_{o}$ and the integral length scale $L_{t}$. Here again, notwithstanding the relatively large scatter, experiments indicate that $\eta_{o}$ is globally proportional to $L_{t}$. In the present case, the coefficient of proportionality is found to be about 3 .

\section{B. Prediction of the fractal dimension $\beta$}

Sreenivasan et al. [29] suggested that at infinite Reynolds numbers, the exponent $\beta$ of a passive interface should be equal to $1 / 3$. For reacting flows, Peters et al. [1] soon realized that the kinematic restoration mechanism might act in smoothing the flame interface and derived an expression highlighting the effect of $u^{\prime} / S_{l}$ on $\beta$, viz. [23]

$$
\beta=\left(3+\frac{1}{u^{\prime} / S_{l}-1}\right)^{-1}
$$

The same year, on the basis of an extensive experimental study, North et al. [18] end up with an em- 
pirical relation of the form

$$
\beta=\frac{2.05}{u^{\prime} / S_{l}+1}+\frac{2.35}{S_{l} / u^{\prime}+1}-2
$$

Chakraborty and Klein [90] also proposed an expression for describing the evolution of $\beta$ with respect to the Karlovitz number

$$
\beta=\frac{1}{3} \operatorname{erf}(K a)
$$

More recently, another Karlovitz-dependent expression for $\beta$ was suggested $[11,20]$

$$
\beta=\frac{1}{3}+\frac{1}{3} \frac{r_{1}^{*} K a^{-1 / 2}}{r_{1}^{*} K a^{-1 / 2}+K a^{-2}}
$$

so that the ratio of the turbulent to laminar flame speed given by $\left(\eta_{o} / \eta_{i}\right)^{\beta}$ is consistent with Damköhler predictions at both high and low Karlovitz numbers. Eq. (30) suggests that $\beta$ is $1 / 3$ at low Karlovitz number and $2 / 3$ in the high Karlovitz regime in agreement with recent findings of DNS at very high Ka [21].

The fractal dimension $\beta$ obtained from our database is compared to that predicted by either Eq. (27), Eq. (28), Eq. (29) and Eq. (30) in Figs. 16 and 17. Despite the scatter, experimental data globally confirm the increasing tendency of $\beta$ with respect to $u^{\prime} / S_{l}$ (Fig. 16). Predictions arising from either Eq. (27) or Eq. (28) are roughly consistent with this increase even though the scatter in the data hampers drawing any firm conclusions. In Fig. 17, experimental values of $\beta$ are compared to Eqs. (29) and (30). It clearly appears that Eq. (30) differs significantly with experiments and that $\beta$ is much closer to a value of $1 / 3$ than $2 / 3$. Here also, the scatter in the data is too large to assess the ability of Eq. (29) for predicting $\beta$.

Both the scatter in the data and the discrepancy with theoretical or empirical predictions are likely to be related to the aforementioned finite Reynolds number effects. Indeed, because of the limited ratio between $\eta_{o}$ and $\eta_{i}$ as emphasized in Fig. 12, the large scales, which are known to be non universal in that sense that they depend on the type of initial and boundary conditions, strongly affects the statistical behaviour of the inertial and small scales. Consequently, much higher values for the logarithmic separation $a$ are needed to draw firm statements about the evolution of $\beta$ with respect to the flow and flame parameters. As shown in Fig. 15, $\eta_{i}$ is proportional to the Kolmogorov length-scale $\eta$, i.e. $\eta_{i} \approx 10 \eta$, whilst $\eta_{o} \approx 3 L_{t}$. Since $R e_{t} \sim\left(L_{t} / \eta\right)^{3 / 4}$, it comes $R e_{t} \approx\left(\frac{10}{3} \frac{\eta_{o}}{\eta_{i}}\right)^{4 / 3}$. Therefore, a clear inertial range, i.e. $\eta_{o} / \eta_{i} \approx 10^{3}$ for $\mathcal{F}_{\max } \approx 1$, might be achieved for $R e_{t} \approx 5.10^{4}$. Assuming that $\eta \sim \delta_{L} \sim 0.1 \mathrm{~mm}$ so that $K a \sim 1$ lo lie within the flamelet regime, then $L_{t}$ should be of the order of $30 \mathrm{~cm}$. This type of turbulence is clearly beyond the reach of existing laboratory experiments. Hence, as it was done in non reacting fluid turbulence, for which the quest for large-scale and very high Reynolds number experiments have motivated many recent studies, similar needs now arise in the field of combustion. As a consequence, before confirming available predictions for $\beta$, a dynamical procedure for estimating this parameter might be preferably invoked in Large Eddy Simulation of turbulent premixed combustion at relatively low Reynolds numbers (see e.g. [10, 11, 24]).

\section{HEURISTIC PHENOMENOLOGY OF WRINKLED INTERFACES}

Heretofore, in this paper, experimental data of the turbulent flames have been analysed with the goal of demonstrating the ability of POD for quantifying their scale-by-scale statistics and the suitability of some theoretical/empirical relations for predicting them. In the following, we now concentrate on the particular phenomenological aspects of flame wrinkling and on the degree of generality that this phenomenology can reveal for giving further insight into the corrugation of some other interfaces.

\section{A. Analytical aspects}

It is well known that the hyperbolic tangent is solution of the differential equation $f^{\prime}+f^{2}-1=0$. The appearance of the hyperbolic tangent in Eq. (19) thus indicates that the scale entropy flux $\mathcal{F}(\mathcal{R})$ is governed by the following differential equation

$$
\frac{1}{2 a} \frac{\partial \mathcal{F}(\mathcal{R})}{\partial \mathcal{R}}-\mathcal{C}(\mathcal{R}) \times \mathcal{F}(\mathcal{R})=0
$$

or equivalently, the differential equation for $\mathcal{L}(\mathcal{R})$ is

$$
\frac{1}{2 a} \frac{\partial^{2} \mathcal{L}(\mathcal{R})}{\partial \mathcal{R}^{2}}-\mathcal{C}(\mathcal{R}) \frac{\partial \mathcal{L}(\mathcal{R})}{\partial \mathcal{R}}=0
$$

where

$$
\mathcal{C}(\mathcal{R})=\frac{1}{2}[\tanh (a \mathcal{R})+\tanh (a \mathcal{R}+a)] .
$$

Even though the analysis is provided here in the context of statistically stationary turbulent flames, the time derivative of $\mathcal{L}$ can be added to Eq. (32) as was done in $[34,35,84]$. In this case, the transport equation for $\mathcal{L}$ writes

$$
\frac{1}{\gamma} \frac{\partial}{\partial t} \mathcal{L}(\mathcal{R}, t)+\mathcal{C}(\mathcal{R}, t) \frac{\partial \mathcal{L}(\mathcal{R}, t)}{\partial \mathcal{R}}=\frac{1}{2 a} \frac{\partial^{2} \mathcal{L}(\mathcal{R}, t)}{\partial \mathcal{R}^{2}},
$$

where $\gamma$ is referred to as a scale-entropy diffusion coefficient [34, 35, 84]. Eq. (34) can be interpreted as a one-dimensional convection-diffusion equation, where the scalar $\mathcal{L}$ depends on space $\mathcal{R}$, the rightmost term of Eq. (34) represents the diffusion mechanism whereas the second term on left hand side corresponds to the transport of $\mathcal{L}$ by a 'convection velocity' $\mathcal{C}(\mathcal{R})$. 
On the other hand, one can also do some algebra and end up with a pure diffusion equation with a source term consistently with [84], or a diffusion/convection equation with a source term. With these different interpretations in mind, it is however worth stressing that keeping this formulation for Eq. (34) allows us to highlights interesting analogies between Eq. (34) and other transport equations which are widely used in the field of turbulence and/or turbulent combustion.

Indeed, one can note that Eq. (34) is analogous to the Lin's equation [92] which describes the transport of velocity energy spectrum $E(q, t)$ in homogeneous isotropic turbulence, viz

$$
\frac{\partial}{\partial t} E(q, t)+T(q, t)=2 \nu q^{2} E(q, t)
$$

where $q$ is the wavenumber and $T(q, t)$ is the energy transfer spectrum. A similar equation holds for the scalar energy spectrum $[86,93]$. The convective term in Eq. (34) thus relates to the transfer term $T(q, t)$ which itself arises from the non-linear convective term in the Navier-Stokes equation. On the other hand, the dissipative term $2 \nu q^{2} E(q, t)$ corresponds to the right hand side of Eq. (34). By further proceeding in the analogy, one notices that the viscosity $\nu$ in Eq. (35) can be identified with $\gamma / 4 a$. There is thus an analogy between $a=\log \left(\eta_{o} / \eta_{i}\right)$ and the Reynolds number in fluid turbulence. For instance, for a given $\gamma$, the weaker is the viscosity in the Lin's equation, the more the logarithmic separation between $\eta_{o}$ and $\eta_{i}$ and vice versa. This is obviously also the case in fluid turbulence where the logarithmic separation between the integral length and the Kolmorogov length-scale is proportional to the Reynolds number.

Note also that Peters [3] has derived a transport equation for the energy spectrum of a propagating interface whose formulation is also analogous to either Eq. (34) or Eq. (35).

Furthermore, by removing the leftmost term in Eq. (19) which is equivalent to focusing only on inertial and small scales, one obtains that the scale entropy $\mathcal{L}$ is solution of the following partial differential equation

$$
\frac{1}{\gamma} \frac{\partial \mathcal{L}}{\partial t}=\frac{1}{2 a} \frac{\partial^{2} \mathcal{L}}{\partial \mathcal{R}^{2}}+\left(\frac{\partial \mathcal{L}}{\partial \mathcal{R}}\right)^{2}-\frac{1}{4}
$$

This equation looks like the Kardar-Parisi-Zhang (KPZ) equation [94]. The KPZ equation is widely used notably for predicting the diffusion-limited growth of rough interfaces [83]. It is therefore not surprising that the latter may also govern the scale entropy. In the field of combustion, the application of the KPZ equation, though not widespread, is not new. It has notably gave significant insight into the prediction of turbulent flame propagation in weak turbulence [95] and the comprehension of slow combustion of solid material [96]. Eq. (36) is also quite analogous to the Kuramoto-Sivashinsky (KS) equation [97, 98] for which the dispersion term would have been omitted. This suggests that Eq. (34) together with Eq. (12) are likely to be demonstrated on some more fundamental basis using for instance the G-equation, the $\mathrm{KPZ}$ or the KS equation. Analytical results for the inner and outer cut-off might be also obtained by use of these equations. This question is beyond the scope of the present study but is worth being revisited in further investigations.

\section{B. Implications}

This analogy between the scale entropy transport equation and the Lin's equation indicates that a rather pragmatic phenomenology for describing the scale-by-scale properties of wrinkled turbulent flames can be invoked. It indeed appears that the wrinkling is driven by two distinct mechanisms, (i) a diffusion process and (ii) transfer of interface between the different scales. In the context of turbulent flames, the destruction of flame surface is related to the socalled kinematic restoration mechanism $[3,4]$, i.e. the smoothing by the effect of the flame propagation velocity. The latter diffusion processes might also be attributed to the viscous effects that damp the turbulence activity at small scales. On the other hand, the transfer of flame interface through the different scales $\mathcal{R}$ is likely to be more complex since it relates to the cascade process inherent to all turbulent flows. In the field of fluid mechanics, this equilibrium assumption between diffusion and transfer was first initiated by the pioneering work of Kolmogorov [68, 69] for describing turbulent flows. Since then, it has become extremely common. It is therefore not surprising to draw similar conclusions for turbulent reacting flows.

The phenomenology described above suggests that the scale entropy formalism together with Eq. (15) for describing $L(r)$ might not be restricted to turbulent flame fronts only. This also transpires notwithstanding the aforementioned analogy between Eq. (34) and the KPZ equation which is reputed to apply in many unrelated problems. Indeed, this phenomenology could a priori hold for describing the scale-by-scale (possibly fractal) properties of some other wrinkled objects as far as they are driven by diffusive and convective processes. These includes (though not limited to) material lines or surfaces evolving in turbulent flows [36, 99, 100], turbulent/non turbulent interfaces [28, 32], two-phase flows (see the rather recent use of the scale entropy formalism made by $[34,35]$ ), or more generally iso-scalars in homogeneous and/or heterogeneous gaz and/or liquid mixtures [101]. Even though further work is needed to confirm this, the formalism employed here and related results (especially Eq. (34) which emanates directly from Eq. (12)) thus appear to be rather heuristic in that sense they are likely to be generalized to some other kind of interfaces. The aforementioned 


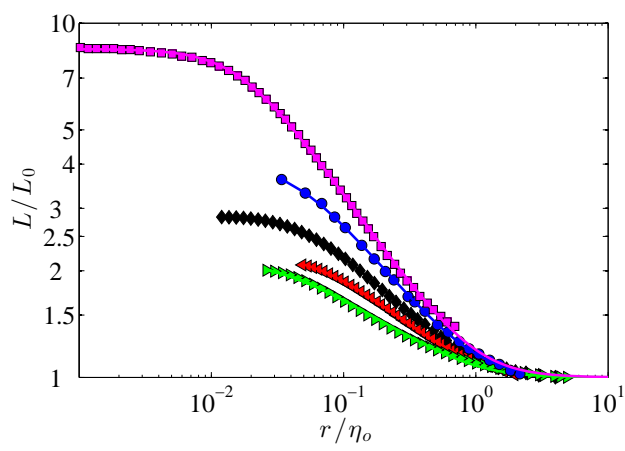

FIG. 18. (Color online). Large-scale similarity. The symbols represent the experimental assessement whereas the lines correspond to the fit using Eq. (12). Black diamonds: Flame 13, Green right triangle: cavitating flow, Red left triangle: iso-scalar, Blue circles: turbulent/non turbulent interface [32], Magenta squares: Two phase flow [102]

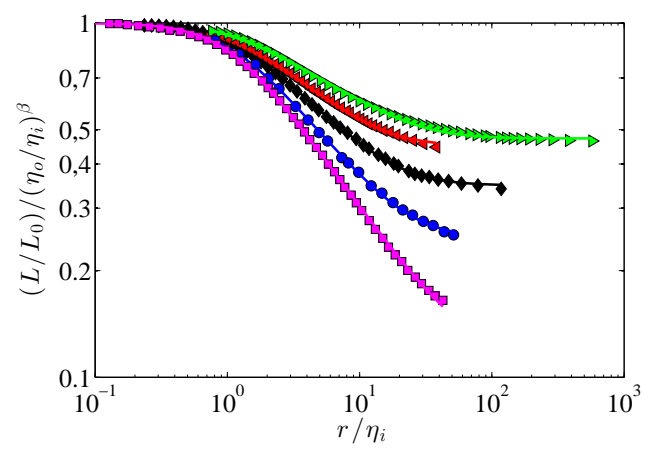

FIG. 19. (Color online). Small-scale similarity. For legend see Fig. 18

different physical situations for which Eq. (34) may apply, are found in many combustion processes. For instance, in rocket engines or direct injection spark ignition engines, the chain of physical processes starting from the atomization of liquid fuel $\rightarrow$ evaporation $\rightarrow$ turbulent mixing $\rightarrow$ diffusion and/or partially premixed turbulent flames could possibly be treated in this context. Hence, our opinion is that the generalization of the scale-entropy formalism to other kind interfaces is worth being mentioned and investigated further in the present paper.

\section{Experimental evidence}

In this purpose, we consider in the following five types of wrinkled interfaces. The POD algorithm was applied to each interface spatial coordinates and the arclength was calculated in a similar fashion as described previously. These interfaces include

(i) A turbulent flame. The Flame 13 of the present database is considered as an example.

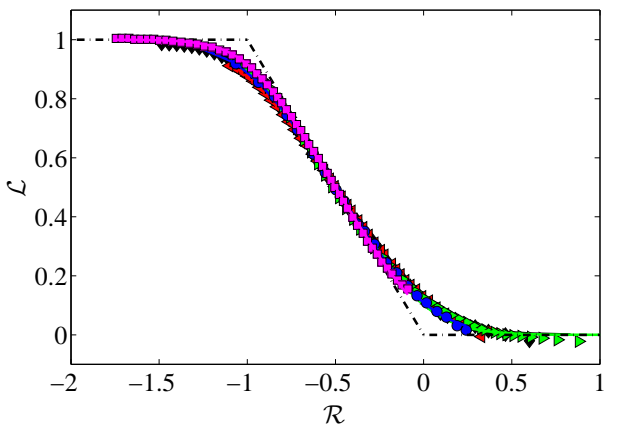

FIG. 20. (Color online). Scale entropy. Symbols and lines are same as Fig. 18

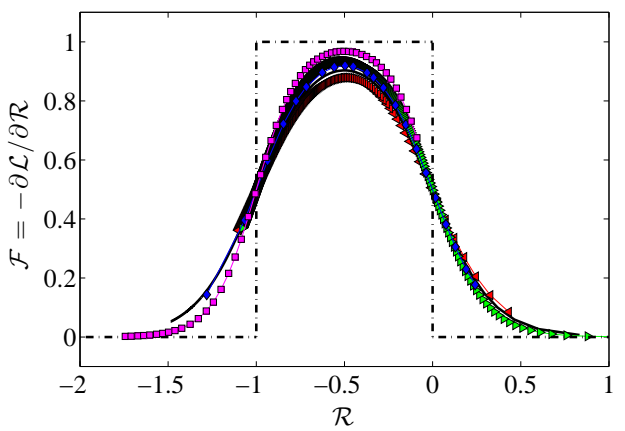

FIG. 21. (Color online). Scale entropy flux. Symbols and lines are same as Fig. 18

(ii) The interface separating the turbulent / non turbulent region in a very high Reynolds number boundary layer. For this particular case, results for $L(r)$ are not obtained by applying the POD algorithm but are taken directly from Fig. 5(b) of [32].

(iii) To be as close as possible from some combustion applications, we also considered a turbulent iso-scalar field evolving in an optically accessible engine [103]. Measurement were done by means of PLIF on acetone which is used as a fuel tracer. The experimental setup is not recalled but the reader can refer to [103] for details. The data reduction for the PLIF images is also fully described in [103].

(iv) The interface separating the liquid to the vapor phase in a cavitating backward facing step flow. Here again, the reader can refer to $[104,105]$ where a detailed description of the experimental setup is provided. Specifically, the case 'cav2' in $[104,105]$ is considered for the present study. The direct visualization of the interface was carried out using a high speed camera. The interface detection was performed using the same procedure as described in $\S \mathrm{II} \mathrm{A}$.

(v) The interface separating the gas to the liquid in a liquid jet destabilized by a surrounding air 
flow. Here also, results for $L(r)$ are not obtained by applying the POD algorithm but are obtained by digitizing the top curve of Fig. 6.16 of [102].

Figs. 18 to 21 depicts the scale-by-scale contribution to the arclength for the four interfaces investigated here. By focusing on Figs. 18 and 19, it clearly appears that Eq. (12) is perfectly tailored for representing $L(r)$, irrespectively of the interface considered. For the iso-scalar data, the arclength has been calculated using different iso-scalar values. Results were qualitatively similar, i.e. Eq. (12) was still perfectly suited for fitting experimental results. The scale entropy and the scale entropy flux are also presented (Figs. 20, and 21). These quantities suggest that even for the boundary layer data for which the Reynolds number was extremely high, or the liquid jet of [102] which provides $\eta_{o} / \eta_{i} \approx 60$, the scale separation between $\eta_{o}$ and $\eta_{i}$ is still not sufficiently large to reach the asymptotic scaling at very large value of $a$. This means that these asymptotic scaling are still beyond the reach of the existing laboratory experiments and that further efforts are needed to attain these extreme regimes.

As a consequence, the fact that the five different interfaces match perfectly with Eq. (12) means that the transport equation Eq. (34) applies as a direct consequence. This is the experimental evidence that the phenomenology described above, emphasizing the primordial role played by diffusion and convection, although not demonstrated by use of either the exact transport equations or simplified stochastic or deterministic equations, is indeed heuristic in that sense that it generalizes to a large number of apparently unrelated physical situations. To our opinion, this might have several useful implications notably for describing and modelling the physical processes at play at the level of these interfaces which are all widely encountered in many practical situations.

\section{SUMMARY}

An extensive experimental database, consisting of 24 turbulent premixed Bunsen flames with 19 independent operating conditions, has been analysed in detail. The flame front is tracked by means of either low or high frame rate Mie scattering tomography and standard binarization procedure. POD is applied to the flame front spatial coordinates with the goal of extracting the geometrical properties of turbulent interfaces. The latter is measured by calculating scale-by-scale contribution to the arclength, i.e. the length of the interface reconstructed using $k$ modes. Special care has been also given to the correspondence between a mode number $k$ and a physical scale $r$. In this context, we proposed a method that revealed that the mode number is linearly related to the wavenumber, i.e. the inverse of the physical scale. Yields the following outcomes

(i) The POD compares favourably well with the caliper technique except at large scales where the latter method is reputed to be biased. For comparing the fractal characteristics of flame wrinkling inferred from these two methods, a parametric expression for $L(r)$ is proposed that allows to compute $\eta_{o}, \eta_{i}$ and $\beta$ in a totally unambiguous fashion. This expression, though not demonstrated, appears to be perfectly tailored for describing $L(r)$ irrespectively of the flame or the interface considered.

(ii) In analogy with non reacting turbulent flows, the degree with which $L(r)$ complies with scalesimilarity is appraised. Three type of similarity are concerned, i.e. large-scale similarity, smallscale similarity and log-similarity. It is observed that none of these three types of similarity holds for the range of Reynolds number investigated here. It is argued that finite Reynolds number effects may be at play and that much higher separations between $\eta_{o}$ and $\eta_{i}$ have to be reached for drawing firm conclusions about the plausibility of scale-similarity for turbulent flames. In this context, the asymptotic approach of $\mathcal{F}_{\max }$ towards unity, which provides an observable of the extent of the inertial range, is discussed in detail.

(iii) The ability of fractal models for predicting the flame surface density is confirmed. The fractal characteristics $\eta_{o}, \eta_{i}$ and $\beta$ are compared to available empirical or phenomenological relations. Whilst the predictions for $\eta_{o}$ and $\eta_{i}$ appears relatively robust, experimental data (especially for $\beta$ ) remain particularly scattered. Here again, it is pleaded that much higher Reynolds numbers are needed before reassessing the evolution of $\beta, \eta_{o}$ and $\eta_{i}$ as a function of the flow and/or flame parameters. For instance, $R e_{t} \approx 5.10^{4}$ may be the limiting value that should be achieved for $\mathcal{F}_{\text {max }}$ to be equal to one within a percent, so that a clear scaling range is likely to be discernible.

(iv) A rather pragmatic phenomenology for the flame wrinkling, which emanates directly from Eq. (12), is finally discussed. A balance equation for the scale entropy $\mathcal{L}(\mathcal{R}, t)$ is obtained, revealing the primordial role played by diffusion and convection in the destruction and/or creation of interface. This equation appears analogous to some exact transport equations such as the Lin's equation (the transport equation of the kinetic energy spectrum), the Corrsin's equation (the transport equation of a passive scalar energy spectrum) or the Peters' equation 
(the transport equation of the interface spectrum). It also reveals some interesting degree of closeness with either the stochastic KPZ or deterministic KS equation. This suggests that Eq. (12) is likely to be demonstrated on some more physical basis. This will be the topic of a future study. It thus transpires that the latter phenomenology could apply to some other folded interfaces as long as they are driven by convective and diffusive processes. It is indeed confirmed by experiments that Eq. (12) is nicely tailored for representing $L(r)$ for the four interfaces considered here. As a direct consequence, Eq. (34) applies to these interfaces. Hence, the scale entropy formalism opens a promising alternative for understanding and modelling the statistical behaviour of corrugated interfaces. To our opinion, this result should lead to important progress since such folded interfaces are widely encountered in many different situations. Specifically, it turns out that in LES, fractal models such as that developed by e.g. $[10,11,20]$ for assessing the unresolved flame surface density can be applied to turbulence, turbulent mixing or two-phase flows.

\section{ACKNOWLEDGEMENTS}

The financial support from the Agence National de la Recherche under the project IDYLLE is gratefully acknowledged. We are thankful to the CNRS, the University of Orléans, and the French Government Program "Investissements d'avenir" through the LABEX CAPRYSSES. FT acknowledges EADS for its financial support. We also benefited from the computing ressources provided by CaSciModOT. We thank Laurent Catherine for his technical assistance.

\section{Appendix A: Determination of the number of modes}

In order to minimize the computational cost of the POD algorithm, one generally sets the number of modes $N_{m}$ as the smallest dimension of $\chi$ i.e. $N_{m}=\min \left\{2 N_{p}, N_{i m}\right\}$. Hence, two cases can be considered depending on $N_{i m}$ and $N_{p}$

(i) $N_{i m}>2 N_{p}$ (high frame rate tomography), in which case the direct method $[39,41]$ is invoked. The correlation matrix $\boldsymbol{R}^{*}$ is then defined as

$$
\boldsymbol{R}^{*}=\chi \chi^{T}
$$

Then, using Eqs. (4) and (6a), it is readily shown that

$$
\boldsymbol{R}^{*}=\chi \chi^{T}=\boldsymbol{\Phi} \boldsymbol{W}^{2} \boldsymbol{\Phi}^{T}=\boldsymbol{\Phi} \boldsymbol{\Lambda} \boldsymbol{\Phi}^{-1} .
$$

One identifies from Eq. (A2) an eigenvalue problem, yields estimation of $\boldsymbol{W}^{2}=\boldsymbol{\Lambda}$, the diagonal eigenvalue matrix and $\boldsymbol{\Phi}$, the eigenvector matrix. Once $\boldsymbol{\Phi}$ is known, the temporal coefficient matrix $\boldsymbol{\Psi}$ is obtained by projecting $\chi$ on the eigenvectors $\boldsymbol{\Phi}$.

(ii) $N_{i m}<2 N_{p}$ (low frame rate tomography), in which case the snapshot method [40] is employed. The correlation matrix $\boldsymbol{R}$ is then defined as

$$
\boldsymbol{R}=\chi^{T} \chi
$$

Plugging Eqs. (4) and (6b) into Eq. (A3), one ends up with

$$
\boldsymbol{R}=\chi^{T} \boldsymbol{\chi}=\boldsymbol{\Psi}^{T} \boldsymbol{W}^{2} \boldsymbol{\Psi}=\boldsymbol{\Psi}^{-1} \boldsymbol{\Lambda} \boldsymbol{\Psi}
$$

Contrary to the previous case, from Eq. (A4) yields the temporal coefficient matrix $\boldsymbol{\Psi}$ and $\boldsymbol{\Lambda}$, and the mode matrix $\boldsymbol{\Phi}$ is obtained by projecting $\chi$ on the eigenvectors $\Psi$
[1] N. Peters, on Symposium
ternational) Symposium (International) on Combustion 21, 1231
D. Veynante and L. Vervisch, Prog. Energ. Combust. 28, 193 (2002).

[3] N. Peters, J. Fluid Mech. 242, 611 (1992).

[4] N. Peters, J. Fluid Mech. 384, 107 (1999).

[5] H. Pitsch and L. Duchamp de Lageneste, Proc. Combust. Inst. 29, 2001 (2002).

[6] S. Pope, Int. J. Eng. Sci. 26, 445 (1988).
[7] K. Bray, M. Champion, and P. A. Libby, in Turbulent Reactive Flows (Springer, 1989) pp. 5411986). 563.

[8] T. Poinsot, S. Candel, and A. Trouvé, Prog. Energ. Combust. 21, 531 (1995).

[9] C. Fureby, Proc. Combust. Inst. 30, 593 (2005).

[10] F. Charlette, C. Meneveau, and D. Veynante, Combust. Flame 131, 181 (2002).

[11] E. R. Hawkes, O. Chatakonda, H. Kolla, A. R. Kerstein, and J. H. Chen, Combust. Flame 159, 2690 (2012). 
[12] F. Thiesset, L. Danaila, and R. A. Antonia, J. Fluid Mech. 720, 393 (2013).

[13] K. R. Sreenivasan and R. A. Antonia, Annu. Rev. Fluid Mech. 29, 435 (1997).

[14] R. Antonia, L. Djenidi, and L. Danaila, Phys. Fluids 26, 045105 (2014).

[15] F. Gouldin, Combust. Flame 68, 249 (1987).

[16] F. Gouldin, S. Hilton, and T. Lamb, Symposium (International) on Combustion, Symposium (International) on Combustion 22, 541

[17] Ö. L. Gülder and G. J. Smallwood, Combust. Flame 103, 107 (1995).

[18] G. North and D. Santavicca, Combust. Sci. Technol. 72, 215 (1990).

[19] C. Cohé, F. Halter, C. Chauveau, I. Gökalp, and Ö. L. Gülder, Proc. Combust. Inst. 31, 1345 (2007).

[20] F. Thiesset, G. Maurice, F. Halter, N. Mazellier, C. Chauveau, and I. Gokalp, submitted to Combustion Theory and Modelling (2015).

[21] O. Chatakonda, E. R. Hawkes, A. J. Aspden, A. R. Kerstein, H. Kolla, and J. H. Chen, Combust. Flame 160, 2422 (2013).

[22] A. R. Kerstein, Combust. Sci. Technol. 60, 441 (1988).

[23] N. Peters and C. Franke, in Dissipative Structures in Transport Processes and Co (Springer, 1990) pp. 40-50.

[24] G. Wang, M. Boileau, D. Veynante, and K. Truffin, Combust. Flame 159, 2742 (2012).

[25] M. Germano, U. Piomelli, P. Moin, and W. H. Cabot, Phys. Fluids 3, 1760 (1991).

[26] T. Ménard, S. Tanguy, and A. Berlemont, Int. J. Multiphase Flow 33, 510 (2007).

[27] A. R. Kerstein, W. T. Ashurst, and F. A. Williams, Phys. Rev. A 37, 2728 (1988).

[28] K. Sreenivasan and C. Meneveau, Journal of Fluid Mechanics 173, 357 (1986).

[29] K. Sreenivasan, R. Ramshankar, and C. Meneveau, Proceedings of the Royal Society of London A: Mathematical, Physical and Engineering Sciences, Proc. Roy. Soc. Lond. A 421, 79 (1989).

[30] K. R. Sreenivasan, Annu. Rev. Fluid Mech. 23, 539 (1991).

[31] H. J. Catrakis and P. E. Dimotakis, J. Fluid Mech. 317, 369 (1996).

[32] C. M. de Silva, J. Philip, K. Chauhan, C. Meneveau, and I. Marusic, Phys. Rev. Lett. 111, 044501 (2013).

[33] L. Le Moyne, V. Freire, and D. Q. Conde, Chaos, Solitons \& Fractals 38, 696 (2008).

[34] C. Dumouchel and S. Grout, Int. J. Multiphase Flow 35, 952 (2009).

[35] C. Dumouchel and S. Grout, Phys. A 390, 1811 (2011).

[36] E. Villermaux and Y. Gagne, Phys. Rev. Lett. 73, 252 (1994).

[37] H. J. Catrakis, R. C. Aguirre, and J. RuizPlancarte, J. Fluid Mech. 462, 245 (2002).

[38] S. Jay, F. Lacas, and S. Candel, Combust. Flame 144, 558 (2006).

[39] J. L. Lumley, Computational modeling of turbulent flows, Advances in Applied Mechanics (Hermessciences, 1978).

[40] L. Sirovich, M. Kirby, and M. Winter, Physics of Fluids 2, 127 (1990).
[41] G. Berkooz, P. Holmes, and J. L. Lumley, Annu. Rev. Fluid Mech. 25, 539 (1993).

[42] J. Borée, Exp. in Fluids 35, 188 (2003).

[43] S. J. Danby and T. Echekki, Combust. Flame 144, 126 (2006).

[44] C. Duwig and C. Fureby, Combust. Flame 151, 85 (2007).

[45] C. Duwig and L. Fuchs, Phys. Fluids 19, 075103 (2007).

6] S. Kostka, A. C. Lynch, B. C. Huelskamp, B. V. Kiel, J. R. Gord, and S. Roy, Combust. Flame 159, 2872 (2012).

[47] G. Maurice, F. Thiesset, N. Mazellier, F. Halter, C. Chauveau, I. Gökalp, and A. Kourta, Exp.Therm. Fluid Sc. In press (2015), 10.1016/j.expthermflusci

[48] C. W. Rowley, I. Mezić, S. Bagheri, P. Schlatter, and D. S. Henningson, J. Fluid Mech. 641, 115 (2009).

[49] P. J. Schmid, J. Fluid Mech. 656, 5 (2010).

[50] N. E. Huang, Z. Shen, S. R. Long, M. C. Wu, H. H. Shih, Q. Zheng, N.-C. Yen, C. C. Tung, and H. H. Liu, Proc. Roy. Soc. London, Proc. Roy. Soc. Lond. A 454, 903 (1998).

[51] P. Flandrin, G. Rilling, and P. Goncalves, Signal Processing Letters, IEEE 11, 112 (2004). $n$ A. Boukhalfa and I. Gökalp, Combust. Flame 73, 75 (1988).

[53] R. Fragner, N. Mazellier, F. Halter, C. Chauveau, and I. Gökalp, Flow, Turbulence and Combustion 84, 25 (2014).

[54] R. Fragner, F. Halter, N. Mazellier, C. Chauveau, and I. Gökalp, Proc. Combust. Inst. 35(2), 1527 (2015).

[55] N. Mazellier, L. Danaila, and B. Renou, Journal of Turbulence 11, 43 (2010).

[56] G. Smith, D. Golden, M. Frenklach, N. Moriarty, B. Eiteneer, M. Goldenberg, C. Bowman, R. Hanson, S. Song, W. Gardiner, et al., GRI-Mechan optimized detailed chemical reaction mechanism for methane combustion, Tech. Rep. (Technical Report http://www. me. berkeley. edu/gri mech, Gas Research Institute, 1999).

[57] R. Borghi, in Recent advances in the aerospace sciences (Springer, 1985) pp. 117-138.

[58] P. Goix, P. Paranthoën, and M. Trinite, Combust. Flame 81, 229 (1990).

[59] A. Lipatnikov and J. Chomiak, Combust. Sci. Technol. 162, 85 (2001).

[60] E. Cintosun, G. Smallwood, and O. Gülder, AIAA Journal 45, 2785 (2007).

[61] G. K. Batchelor, Proc. Camb. Phi. Soc. 47, 359 (1951).

[62] G. Stolovitzky, K. R. Sreenivasan, and A. Juneja, Phys. Rev. E 48, R3217 (1993).

[63] S. Kurien and K. R. Sreenivasan, Phys. Rev. E 62, 2206 (2000).

[64] K. G. Aivalis, K. R. Sreenivasan, Y. Tsuji, J. Klewicki, and C. A. Biltoft, Phys. Fluids 14, 2439 (2002).

[65] R. A. Antonia, R. J. Smalley, T. Zhou, F. Anselmet, and L. Danaila, J. Fluid Mech. 487, 245 (2003).

[66] R. A. Antonia and P. Burattini, J. Fluid Mech. 550, 175 (2006).

[67] F. Thiesset, R. A. Antonia, and L. Danaila, Phys. Fluids 25(11), 115105 (2013).

[68] A. Kolmogorov, Proc. USSR Ac. of Sci. 30, 299 (1941). 
[69] A. Kolmogorov, Dokl. Akad. Nauk. SSSR 125, 15 (1941).[88] W. L. Roberts, J. F. Driscoll, M. C. Drake, and

[70] B. Mandelbrot, in Logique, langage et théorie de l'information (Paris, Presses universitaires de France, 1957) pp. 22-53.

[71] J. Rigaut, J. Microsc. 133, 41 (1984).

[72] J. Rigaut, in Fractals : Non integrable dimensions and applications (G. Cherbit, Ed., (Wiley, Chichester, UK, 1991), 1991) pp. 151-187.

[73] B. Mandelbrot, The fractal geometry of nature, Vol. 1 (W. H. Freeman, New York, 1982) p. 495 p.

[74] Y.-C. Chen and M. Mansour, Exp. in Fluids 26, 277 (1999).

[75] S. Daniele, J. Mantzaras, P. Jansohn, A. Denisov, and K. Boulouchos, J. Fluid Mech. 724, 36 (2013).

[76] F. Thiesset, V. Schaeffer, L. Djenidi, and R. Antonia, Phys. Fluids 26, 075106 (2014).

[77] X.-Z. Wu, L. Kadanoff, A. Libchaber, and M. Sano, Phys. Rev. Lett. 64, 2140 (1990).

[78] M. Nelkin, Phy. Rev. A 42, 7226 (1990).

[79] U. Frisch and M. Vergassola, in New Approaches and Concepts in Turbulence (Springer, 1993) pp. 29-34.

[80] B. Castaing, Y. Gagne, and M. Marchand, Physica D: Nonlinear Phenomena 68, 387 (1993).

[81] P. Meakin, Progress in Solid State Chemistry 20, 135 (1)

[82] P. Meakin, Physics Reports 235, 189 (1993).

[83] T. Vicsek, Fractal growth phenomena, Vol. 2 (World Scientific, 1992).

[84] D. Queiros-Conde, Proceedings of the Royal Society of London A: Mathematical, Physical and Engineering Sciences, Proc. Roy. Soc. Lond. A 459, 3043 (2003).

[85] L. Mydlarski and Z. Warhaft, J. Fluid Mech. 320, 331 (1996).

[86] L. Danaila and R. A. Antonia, Phys. Fluids 21, 111702 (2009).

[87] F. Halter, C. Chauveau, I. Gökalp, and D. Veynante, Combust. Flame 156, 657 (2009).
L. P. Goss, Combust. Flame 94, 58 (1993).

[89] H. Kobayashi, T. Kawahata, K. Seyama, T. Fujimari, and J.-S. Kim, Proc. Combust. Inst. 29, 1793 (2002).

[90] N. Chakraborty and M. Klein, Phys. Fluids 20, 085108 (2008).

[91] Y. Shim, S. Tanaka, M. Tanahashi, and T. Miyauchi, Proc. of the Comb. Inst. 33, 1455 (2011).

[92] T. von Kármán and C. C. Lin, Advances in Applied Mechanics 2, 1 (1951).

[93] S. Corrsin, J. Appl. Phys. 22, 469 (1951).

[94] M. Kardar, G. Parisi, and Y.-C. Zhang, Phys. Rev. Lett. 56, 889 (1986).

[95] A. R. Kerstein and W. T. Ashurst, Phys. Rev. Lett. 68, 934 (1992).

[96] J. Maunuksela, M. Myllys, O.-P. Kähkönen, J. Timonen, N. Provatas, M. Alava, and T. Ala-Nissila, Phys. Rev. Lett. 79, 1515 (1997).

[97] Y. Kuramoto, Prog. Theor. Phys. Supplement 64, 346 (1978).

[98] G. I. Sivashinsky, Annu. Rev. Fluid Mech. 15, 179 (1983).

[99] P. Yeung, S. Girimaji, and S. Pope, Combust. Flame 79, 340 (1990).

[100] S. Goto and S. Kida, J. Fluid Mech. 586, 59 (2007).

(19001)] P. E. Dimotakis and H. J. Catrakis, in Mixing (Springer, 1999) pp. 59-143.

[102] P. Marmottant, Atomisation d'un liquide par un courant gazeux, Ph.D. thesis, Institut National Polytechnique de Grenoble (2001).

[103] V. M. Salazar, S. A. Kaiser, and F. Halter, Optimizing precision and accuracy of quantitative plif of acetone Tech. Rep. (SAE Technical Paper, 2009).

[104] G. Maurice, H. Djeridi, and S. Barre, in IOP Conference Series: Earth and Environmental Science, Vol. 22 (IOP Publishing, 2014) p. 052008.

[105] G. Maurice, Étude expérimentale de l'interaction turbulence cavitation dans un écoulement de marche descendante cavitant: Application à la problématique du "backflow" à l'amont des turbopompes de moteurs de fusées, Ph.D. thesis, University of grenoble (2014). 